\title{
Conjugate Residual Methods for Almost Symmetric Linear Systems ${ }^{1}$
}

by

Juan Camilo Meza

Technical Report 86-9, April 1986

${ }^{1} \mathrm{~A}$ Thesis submitted in partial fulfilment of the requirements for the degree of Doctor of Philosophy, Rice University. 

RICE UNIVERSITY

CONJUGATE RESIDUAL METHODS

FOR ALMOST SYMMETRIC LINEAR SYSTEMS

by

\section{JUAN CAMILO MEZA}

A Thesis Submitted

In Partial Fulfillment of The

Requirements For The Degree

\section{DOCTOR OF PHILOSOPHY}

Approved, Thesis Committee:

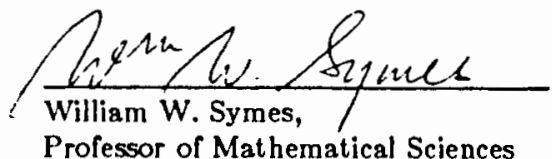

Professor of Mathematical Sciences

Chairman

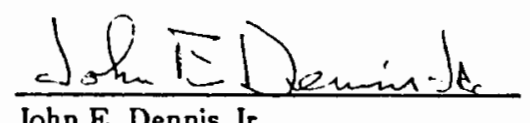

Professor of Mathematical Sciences

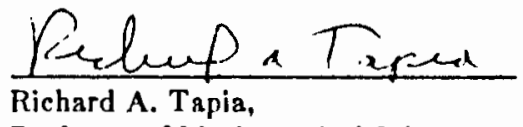

Professor of Matbematical Sciences

$$
\text { Mros Hell }
$$

N. Ross Hill,

Associnte Professor of Geophysics

\section{CONJUGATE RESIDUAL METIIODS}

FOR

\section{ALMOST SYMMETRIC LINEAR SYSTEMS}

\section{JUAN CAMILO MEZA}

\section{ABSTRACT}

This study concerns the use of conjugate residual methods for the solution of nonsymmetric linear systems arising from seismic inverse problems. We focus on an application which has two distinguishing features. The first feature is that the linear system is not readily available. The second feature is that the linear system is almost symmetric. We state and prove a new convergence theorem for a class of Generalized Conjugate Residual methods which shows that in some cases the perturbed symmetric problem can be solved with an error bound similar to the one for the symmetric case.

Houston, Texas

May, 1986 
I would like to thank my committee members John Deanis, ltess Ilill, Hill Symes, and Richard lappia for their help througloout my graduate career. I would especially like to thank my advisor Bill Symes, whose ideas and patience were invaluable. Ny thanks also go to thgram Olkin who carefully real severial drafts of this thesis, and whose timely advice motivated me to tinish. I would also like to thank Anna Apanel, Dan Woods, Maria Rosa Colis, and Mike Pearlman who each in lheir own way provided help, and inspiration when lhe going gol rough. To my parents, Carmen and Camilo, and my entire family, who were alway's there when I needed then, I dedicate this work to you. Finally, I would like to thank my wife Julia who put up with me through so mueh even in the midst of her own thesis work - we made it!

This work was supported in part by NSF grant DMS-8.103148, and in part Ly NSF grant DClR81-16770.
Chapter 1 Introduction

1.1 Statement of the Problem

1.2 Motivation

1.3 Goals

\section{Chapter 2 Preliminaries}

2.1 Notation

2.2 Basic Linear Algebra Theory

2.3 Iteralive Methods

Chapter 3 Krylov Space Methods

3.1 Generalized Conjugate Residual Methods

3.2 Restarled and Truncated Methods

3.3 Convergence Results

Chapter 4 The Nonsymmetric Problem

4.1 Previous Work

4.2 Convergence Analysis for the Symmetric Problem

4.3 Perturbational Anulysis for GCR

4.4 Special Distributions of Eigenvalues

Chapter 5 Numerical Results

5.1 Numerical Examples for Small Perturbations

Chapter 6 Conclusions

Bibliography
10

1

18

18

20 


\section{LIST OF TA TLES}

Clinpter 3 Krylov Space Methods

3.1. GCR work and stornge requirements.

\section{Chapter 4 The Nonsymmetric Problem}

4.1. Chebyshev Coefficients.

4.2. Error term $\tau_{k}$ for $\delta=10^{-6}$.

4.3. Error term $\tau_{k}$ for $\delta=10^{-3}$.

4.4. Error term $\tau_{k}^{k}$ for $\delta=10^{-1}$

\section{Chapter 5 Numerical Results}

5.1. Perturbation to Identity, $\mathrm{N}=5$.

5.2. Perturbation to Identity, $\mathrm{N}=50$.

5.3. Two Clusters at $\lambda_{n}=.1, \lambda_{1}=1.0$. $; \mathrm{N}=50$.

5.4. Two Clusters at $\lambda_{n}=.01, \lambda_{1}=1.0$. $N=50$.

5.5. Two Clusters at $\lambda_{n}=.001, \lambda_{1}=1.0$. $; N=50$.

5.8. Predicted Number of Iterations for the 2 Cluster Cases.

5.7. Three Clusters at $a=0.1, b=0.5, c=1.0 ; N=9$.

5.8. Three Clusters at $\mathrm{a}=0.1, \mathrm{~b}=0.5, \mathrm{c}=1.0 ; \mathrm{N}=30$.

5.9. Uniformly Spaced Eigenvalues $\in\{1.0,10.0\} ; N=10$.

5.10. Uniformly Spaced Eigenvalues $\in[1.0,10.0 \mid ; N=50$.

5.11. Uniformly Spaced Eigenvalues $\in[1.0,100.0\} ; N=50$.

5.12. One Isolated Large Eigenvalue at $\lambda=100 ; N=10$.

5.13. Jordan Blocks, Small $\alpha$.

5.14. Jordan Blocks, $\alpha=1$.

5.15. Spectral Properties of Jordan Blacks.

\section{CHAP'TER}

\section{Introduction}

\subsection{Statement of the Problem}

This study concerns the use of conjugate residual methods for the solution of almost symmetric linear systems such as those arising from seismic inverse problems. The conjugate residual method was originally developed for symmetric positive definite systems, and is usually both efficient and effective over a wide range of problems. Many important physical problems, however, give rise to nonsymmetric linear systems (see Concus and Golub (1976), Vinsome (1976), Symes (1982)). In this study, we focus on an application arising from a seismic inverse problem which has two distinguishing features. The first feature is that the linear system is not readily available. This means that for most practical problems we must resort to an iterative procedure. The second feature is that the linear systern arising from the seismic inverse problem is nearly symmetric.

Many authors have attempted to generalize the conjugate gradient methods to nonsymmetric systems. One such example is the class of Generalized Conjugate Residual (GCR) methods suggested by Eisenstat, Elman, and Schultz (1983). They prove convergence, along with a rate of convergence, for these 
unelhods. The convergence rate derived for the GCR methods is similar to the convergence race for sleepest descent, which can be considerably slower than the rate for the conjugate gradient methods. Since the uonsymmetries in our applicalion are small, il secms plausible that the convergence rate for the nowsymetric conjugate gradient metlods might be similar to the convergence rate for the symmetric problem. In this study, we state and prove a new convergence theorem for a class of GCR methods which shows that in some casey the perturbed symmetric problem can be solved with an error bound similar to the one for the symmetric case.

\subsection{Motivation}

The velocity inversion problem is a member of a class of problems known as seismic inverse problems. The idea behind the seismic inverse problem is to determine a set of parameters describing a mediun, such as the earth, from another set of data usually given on the boundary of the medium. A typical example is the exploration for oil whereby small charges of explosives are set off near the ground and the resulting echoes are recorded at receivers placed near the surface at certain distances away from the explosion. The object of the seismic experiment is to determine a set of paraneters that describe the structure of the earth from the data taken at the receivers.

By the velocity inversion problen we mean the problem of determining the sound speed structure of a medium from its response to an energy source.
Consider the one-dimensional velocily model:

$$
\begin{aligned}
\left(\frac{1}{c^{2}(z)} \partial_{t}^{2}-\partial_{\imath}^{2}\right) u & =0, & & >0, \\
c(0) d, u & =-f(\ell), & z & =0, \\
u & \equiv 0, & & >0, \iota<0 .
\end{aligned}
$$

llere $c(z)$ is the wave speed, $f(l)$ is a source wavelet, and $u(z, t)$ is the wavefield. In our example $f(t)$ is the energy source, that is, the explosion. The wavefield $u(z, \ell)$ may be thought of as displacenent or pressure. In lhis study we assume $f(t)$ is given and that $c(0)$ is known from measurements taken near the surface.

Define a seismogram by

$$
s(t)=\left.\frac{\partial u(z, t)}{\partial t}\right|_{z=0} .
$$

The seismogram may be thought of as the pressure or displacement measured at the receivers after the explosive charge is set off. Notice that every quantity in the boundary value problem (1.2.1) is fixed, except for $c(2)$, so that if the wave speed is varied then the wavefield $u(z, t)$ clianges. Since the seismogram depends on $u(z, t)$, it may be regarded as a function of $c$, that is,

$$
\varepsilon=F(c)
$$

The relation (1.2.2) is known as the forward problem. By the inverse problem we mean the problem of deternining $c$ given a seismogram 8 .

As in most plyysical experiments, the data is known to have some noise. Under these conditions it is unlikely that we can fit the data exactly. Instead 
we consider the least squares problem.

$$
\min \|s-F(c)\|^{2} \text {. }
$$

This is a nonlinear least squares problem. A natural choice to consider for solving this problem is some type of Newton method. For example consider the Gauss-Newton method

$$
J^{*} J \cdot \delta c=-J^{*}(F(c)-8)
$$

where $J=D F(c), J^{*}$ is the adjoint of $J$ :

$$
\left\langle J^{*} \cdot x, y\right\rangle=\langle x, J \cdot y\rangle
$$

and $\langle x, y\rangle$ denotes the $L^{2}-$ inner product. In order to calculate a GaussNewton step it is necessary to compute the actions of $J$ and $J^{*}$ on vectors. Symes (1885) shows that the action of $J$ on a vector is given by

$$
J(c) \cdot \delta c=\langle D F(c) \cdot \delta c, F(c)-8\rangle
$$

The gradient $D F(c) \cdot \delta c$ may be computed from the solution of the perturbational problem

$$
\begin{aligned}
\left(\frac{1}{c^{2}(z)} \partial_{l}^{2}-\partial_{z}^{2}\right) \delta u & =\frac{2 \delta c}{c^{3}} \frac{\partial^{2} u}{\partial t^{2}} \\
\partial_{z} \delta u & \equiv 0, \quad z=0, \\
\delta u & \equiv 0, \quad t<0,
\end{aligned}
$$

where $u(z, t)$ solves the boundary value problem (1.2.1). The gradient is then computed by

$$
D F(c) \cdot \delta c=\left.\frac{\partial \delta u}{\partial t}\right|_{z=0}
$$

The adjoint is calculated by a similar process.

Two remarks are in order. The first remark is that $J \cdot \delta c$ is defined by the solution of a boundary value problem. The second remark is that each evaluation of $J \cdot \delta c$ is subject to a certain amount of discretization error. Both of these remarks also apply to the computation of the action of $J^{\bullet}$ on a vector.

Let us consider the consequences of the second remark. Assume that the boundary value problem (1.2.1) is discretized on a rectangular grid and solved by a finite-difference method. Let the matrix $A$ denote a discretization of $J$, and let the matrix $\tilde{A}$ denote a discretization of the adjoint $J^{*}$. Then we can write the discretized version of equation (1.2.4) as

$$
\tilde{A} A x=\tilde{A} b .
$$

where $A$ is an $m \times n$ matrix, $b$ is an m-dimensional vector, and $x$ is $n n$ dimensional vector. Depending on the discretization used, both $m$ and $n$ can be very large. Typical values are $m=10,000$, and $n=5,000$.

Notice that equation (1.2.4) yields a symmetric positive definite system. However, neither the matrix $A$ nor the matrix $\tilde{A} A$ is readily available, since the action of $A$ on a vector must be computed from the solution of a boundary value problem. Moreover, given the size of a typical problem, computing either matrix by using a set of basis vectors is entirely out of the question. Therefore direct methods for the solution of the discretized version of (1.2.4) can be ruled 
out.

Among the iterative methods available, the conjugate gradient algorithm proposed by Hestenes and stiefel (1052) is a popular method for symmetric positive delinite systems. This approach also has the advantage that we do not have to access the elements of the matrix A directly.

Unfortunately, the discretized equation (1.2.7) is not symmetric. When we discretize both $J$ and $J^{*}$, we cannot hope to satisfy the adjoint relation (1.2.5) exactly for the operators $A$ and $\tilde{A}$, since the discretization errors generated by the computation of $A$ and $\tilde{A}$ are independent. If $(x, y)$ denotes the standard $l_{2}$ inner product, then

$$
(\tilde{A} x, y) \neq(x, A y)
$$

that is, $\tilde{A} \neq A^{T}$. We can model this discretization error by the system

$$
\tilde{N} x=\tilde{b}
$$

where

$$
\begin{gathered}
\tilde{N}=\left(A^{T}+E^{T}\right) A \\
\tilde{B}=\left(A^{T}+E^{T}\right) b,
\end{gathered}
$$

and the matrix $E$ can be thought of as noise generated by the calculation of $A^{T}$. The matrix $E$ is unrelated to the matrix $A$ so that $\bar{N}$ is nonsymmetric. Notice that for simplicity we have chosen to model the perturbed system as if the discretization error aruse from the computation of $A^{T}$.
At first glance, it appears that if the discretization errors are small then the behavior of the conjugate gradient method for this problcm might be similar to that for the symmetric problem. Unfortunately this is not the case. Symes (1082) has shown that even for small discretization errors, the standard conjugate gradient method applied to equation (1.2.7) may diverge. An explunation of this behavior was provided by Dennis (1884). It is well-known that the conjugate gradient method may be viewed as a minimization algorithm applied to a certain quadratic functional. The eonjugate gradient method minimizes this functional by computing a search direction and taking a step along this direction. In this application the search direction depcnds on the vector $A^{T} x$. Since the calculation of $A^{T} x$ is contaminated by noise generated in the discretization procesy the search direction computed by the conjugate gradient method may not be a descent direction. Moreover, using the standard formulas for the conjugate gradient method (Hestents and Stiefel (1052)) the steplength will be positive, so that the new iterate must increase the function value. Therefore, the sequence of iterates generated by the conjugate gradient method on this problem is not guaranteed to converge to the ninimizer, and worse the iterates may diverge. This suggests that we use a nonsynmetric version of the conjugate gradient mothod.

Many authors have worked on the problem of generalizing the conjugate gradient method for nonsymmetric systems. llowever, much of this work has been in the field of elliptic equations, especially those problems arising in 
reservoir engincering. Our application is different. The discretization errors can be adjusted depending on how accurately we solve the various boundary value problems. Therefore, even though the problem is nonsymmetric, it is best thought of as a small perturbation of a symmetric operator.

In Chapter 2, we define the notation used and review the basic linear algebra theory necessary in this study. Chapter 3 introduces Krylov space methods for the solution of linear systems. An example of such a method is the class of Generalized Conjugate Residual (GCR) methods, proposed by Eisenstat, Elman and Schultz (1983). Among these methods, the truncated and restarted versions of GCR are discussed. In Section 3.4 we present some of the convergence theorems for these methods proved by Eisenstat, Elman and Schultz. The nonsymmetric problem is discussed in Chapter 4. We briefly review this field and present the main result of this study in Section 4.3. We show that the GCR method converges with a bound which deviates from the error bound for the symmetric case by a term which depends on the size of the nonsymmetry. An application of the main result for the restarted version of the GCR method is also presented. Several other applications for special distributions of eigenvalues are presented in Section 4.4. In Chapter 5 we present some numerical results for test problems dealing with small perturbations to a symmetric operator. Chapter 6 contains some concluding remarks.

\subsection{Goals}

In this study, we investigate the behavior of conjugate residual methods for the solution of almost symmetric linear systems such as those arising from the velocily inversion problem, with particular emphasis on the following factors:

1) Robust modifications to conjugate residual methods in the presence of small errors.

2) Generalizations of the Chebyshev analysis.

3) A better understanding of the behavior of nonsymmetric conjugate residual methods for nearly symmetric problems. 


\section{CHAL'TEIR}

\section{Notation and l'reliminarieo}

This chapter deals with notation and preliminaries used in this scudy. Section 2.1 introduces the notation. In Section 2.2 we briefly review the basic linear algebra theory necessary in this study. Section 2.3 discusses iterative methods for the solution of linear systerns. Matrix polynomials, which are used extensively in later chapters, are also introduced in this section.

\subsection{Notation}

Lel $x$ and $y$ be real $n$ vectors, und let $A$ be au $n \times n$ real matrix. By $(x, y)$ we mean the standard $l_{2}$ inner product. The $l_{2}$ norm is defined by

$$
\|x\|_{2}=(x, x)^{\mu}
$$

The set of eigenvalues, $\lambda(A)=\left\{\lambda_{1}(A), \cdots, \lambda_{n}(A)\right\}$, of a matrix $A$ are the $\mathrm{a}$ roots of the characteristic equation, $|A-\lambda I|=0$, of $A$. Eigenvalues are ordered

$$
\left|\lambda_{1}\right| \geq\left|\lambda_{2}\right| \geq \cdots \geq\left|\lambda_{n}\right|
$$

The spectral radius, $p(A)$ of an $n \times n$ matrix $A$ is defined by

\subsection{Basic Linear Algebra Theory}

Much of the theory in this study revolves around symmetric positive definite matrices. A symmetric matrix satisties the equation $A=A^{r}$. The matrix $A$ is said $w$ be positive delinite if

$$
(x, A x)>0 \text { for all } x \neq 0 .
$$

For nonsymmetric matrices we detine the splitling

$$
A=M-R,
$$

where

$$
\begin{aligned}
& M=\frac{1}{2}\left(A+A^{T}\right), \\
& R=-\frac{1}{2}\left(A-A^{T}\right)
\end{aligned}
$$

The matrix $M$ is called the symmetric part of $A$; the matrix $R$ is called the skew-symmetric part of $A$. Many of our proofs require that the symmetric part of $A$ be positive definite.

The condition of a malrix turns out to be an important concept. By an illconditioned matrix we mean a matrix where small changes in $x$ may cause large changes in the product $A x$. For any vector norm $\|\cdot\|$, deline a corresponding matrix norm by

$$
\|A\|=\sup _{s \neq 0} \frac{\|A x\|}{\|x\|} \text {. }
$$


In particular, it can be shown ( sec Noble and Daniel (1977) p. 442 ) that for the

Euclidean vector norm $\|\cdot\|_{2}$, the corresponding matrix norm is

$$
\|A\|_{2}=\sqrt{\max \lambda\left(\Lambda^{T} A\right)}
$$

Unless otherwise stated we will just write $\|A\|$ to denote the Euclidean matrix norm. The condition number, $\kappa(A)$, of a matrix $A$ can now be defined by

$$
\kappa(A)=\|A\| \cdot\left\|A^{-1}\right\| .
$$

If the matrix is symmetric then it is straightforward to show that

$$
\kappa(A)=\frac{\left|\lambda_{1}(A)\right|}{\left|\lambda_{n}(A)\right|}
$$

\subsection{Iterative Methods}

Consider the system of iinear equations

$$
\boldsymbol{A} \boldsymbol{x}=\boldsymbol{b} \text {. }
$$

Techniques for solving this system of linear equations are usually classified as either direct or iterative methods. $\Lambda$ direct method is one which guarantees a solution to equation (2.2.1) in a finite number of operations. The number of operations depends on the size of the system. If the matrix $A$ is large then direct methods tend to take considerable time and storage. This may be reduced when the matrix $A$ has a special structure, in which erse, special direct methods may take advantage of the particular structure ( see Duff (1977)). Regardless of the size or structure however, direct methods always assume that the cocficients of the matrix $A$ are available. This is not the case in our application.

In our application, the entries of the matrix $A$ are not readily available. However, we can compute the action of the matrix $A$ on a vector by solving a boundary value problem. This leads us into the area of iterative methods. By an iterative method we mean any method which generates a sequence of approximations to the solution of equation (2.2.1). Iterative methods have the advantage that they do not require that the matrix $A$ be stored. The disadvantage is that they may converge slowly or may even diverge for some applications. In particular, we are interested in polynomial-based iterative methods. These methods generate a sequence of iterates of the form

$$
x_{k}=x_{0}+P_{k}(A)\left(x-x_{0}\right)
$$

where $P_{k}(A)$ is a polynomial in the matrix $A$ of degree at most $k$. If we denote the residual $r_{k}$ by

$$
r_{k}=b-A x_{k},
$$

then equation (2.2.2) is equivalent to

$$
r_{k}=Q_{k}(A) r_{0}
$$

Here $Q_{k}(A)$ is a polynomial in the matrix $A$ of degree at most $\mathrm{k}$, such that $Q_{k}(0)=1$.

One important and useful fact about matrix polynomials is their behavior under orthogonal transformations. For any matrix polynomial $P_{k}(1)$, and any orthogonal matrix $Q$, if $A=Q^{T} T Q$, then 


$$
P_{k}(A)=Q^{T} P_{k}(T) Q
$$

If the matrix $A$ is symactric then it may be diagonalized by an orthogonal matrix so that $T-\operatorname{ding}\left(\lambda_{1}, \cdots, \lambda_{n}\right)$. As a consequence equation $(2.2 .5)$ simplities to

$$
P_{k}(A)=Q^{T} \operatorname{diag}\left(P_{k}\left(\lambda_{1}\right), \cdots, P_{k}\left(\lambda_{n}\right)\right) Q
$$

In other words the inatrix polynomial in $A$ is reduced to a polynomial in the real variables $\lambda$.

Iterative methods require a stopping rule. Usually a measure is defined in terms of how close the approximation is to the solution; the method terminates when this measure is small. We discuss two neasures commonly used in the liceralure.

For a symmetric and posilive definite matrix $A$, deline the error functional

$$
E_{1}\left(x_{k}\right)=\left(x-x_{k}, A\left(x-x_{k}\right)\right)^{h} \equiv\left\|x-x_{k}\right\|_{A},
$$

where $\mathrm{x}$ is the solution to the linear system (2.2.1). Although this appears to be a reasonable measure of the error, it suffers from two deficiencies. The first is that, in general, we do not know what the solution $x$ is. The second is that the $A$-norm is only valid when the matrix $A$ is positive definite. However, $E_{1}$ will be used in some of our convergence analyses.

A secoml measure is based on the error functional

$$
E_{2}\left(x_{k}\right)=\left(A\left(x-x_{k}\right), A\left(x-x_{k}\right)\right)^{4}=\left\|b \cdot A x_{k}\right\|_{2}
$$

This error functional is more practical for many iterative procedures since the residual is already computed. This measure is also used in our convergence analysis. 


\section{CHAPTER}

\section{Krylov Space Methods}

This chapter introduces Krylov space methods for the solution of linear systems.

Section 3.1 defines a Krylov space method. In Section 3.2 , we discuss the Generalized Conjugate Residual (GCR) algorithm and present some of its basic properties. Section 3.3 discusses two modifications to the GCR algorithm called truncated and restarted methods. In the last section of this chapter, we review some of the convergence results for the GCR methods.

\subsection{Generalized Conjugate Residual Methods}

Consider the system of linear equations

$$
A x=b \text {, }
$$

where $x$ and $b$ are $n$ dimensional vectors and $A$ is an $n \times n$ real matrix. If the matrix $A$ is large and sparse then this system is often solved by iterative procedures. In this chapter we present several methods proposed by Eisenstat, Elman, and Schultz (1083), which are in the class of Krylov space methods.

By a Krylov space we mean the vector space defined by

$$
\kappa(v, A, k)=\operatorname{span}\left\{v, A v, \cdots, A^{k-1} v\right\}
$$

A Krylov space method is an iterative method that approximates the solution to equation (3.1.1) by generating iterates of the form

$$
x_{k} \in x_{0}+\kappa\left(r_{0}, A, k\right),
$$

where $x_{0}$ is an initial point, and $r_{0}$ is its corresponding residual.

There are many examples of Krylov space methods in the literature (for a survey see Saad (1985)). We concentrate on a particular class of methods, namely the Generalized Conjugate Residual (GCR) methods. In the following discussion, we now assume that the symmetric part of $A$ is positive definite.

Eisenstat, Elman, and Schultz (1983) suggest the following class of descent algorithms for the solution of equation (3.1.1).

\section{Algoritim 3.1. Generalized Conjugate Residual Method}

$$
\begin{aligned}
& \text { Choose } x_{0} \\
& \text { Compute } r_{0}=b-A x_{0} \\
& \text { Set } p_{0}=r_{0} \\
& \text { For } i=0,1, \cdots \\
& \qquad \begin{array}{r}
a_{i}=\frac{\left(r_{i}, A p_{i}\right)}{\left(A p_{i} A p_{i}\right)} \\
x_{i+1}=x_{i}+a_{i} p_{i} \\
r_{i+1}=r_{i}-a_{i} A p_{i}
\end{array}
\end{aligned}
$$

Compute $p_{i+1}$

The particular choice of $a_{i}$ is one that minimizes $\left\|r_{i+1}\right\|_{2}$ as a function of a so 
that $\left\|r_{1}\right\|_{2}$ decreases at each iteration.

There are differeat versions of this algorithm; these vary in bow the new direction, $p_{1,1}$, is computed. If we impese the condition that

$$
\left(A p_{1}, A p_{j}\right)=0 \text { for } i \neq j \text {. }
$$

then al each iteration $x_{i+1}$ minimizes the residual over the atline space $x_{0}+\left\langle p_{0}, \cdots, p_{1}\right\rangle$. Any set of vectors which satisfy condition (3.1.2) are said wo be $A^{T} A$-conjugate. Condition (3.1.2) leads to the following formulas:

$$
\begin{aligned}
& p_{i+1}=r_{i+1}+\sum_{j=0}^{i} b_{j}^{(i)} p_{j}, \\
& b_{j}^{(i)}=\frac{-\left(A r_{i+1}, A p_{j}\right)}{\left(A p_{j} A p_{j}\right)}, \quad j=0,1, \cdots, i .
\end{aligned}
$$

The algorithin requires storage for the solution vector $x$, the residual $r$, the vector $A r$, and $2(i+1)$ additional vectors for $p$ and $A p$, where $i$ is the iteration number. The vectors $A r_{i+1}$ and $A p_{i+1}$ can share storage thereby reducing the wotal storage to $2(i+1)+2$ vectors of length $n$. The work requirenents are $|3(i+1)+4| n$ multiplications plus 1 matrix vector multiplication per iteration. It is thus apparent that as $i$ increases the method requires a large anount of storage and computations.

\subsection{Restarted and Truncated Methods}

As noted in Section 3.1, the GCR method becomes expensive as the iteration proceds. At each iteration we must orthogonalize the new direction aguinst every previous direction. To overcome this dilficulty, we could orthogonalize the new direction against some small number of previous directions. This can be accomplished using a variety of different methods.

One alternative is to orthogonalize the current direction against the last $k$ directions. We refer to any such method as a truncaled method.

The formulas for the direction vecturs are given by:

$$
\begin{gathered}
p_{i+1}=r_{i+1}+\sum_{j=i-k+1}^{i} b_{j}^{(i)} p_{j}, \quad i=0,1, \cdots \\
b_{j}^{(i)}=\frac{-\left(A r_{i+1}, A p_{j}\right)}{\left(A p_{j}, A p_{j}\right)}, \quad j=i-k+1, \cdots, i .
\end{gathered}
$$

Another method for saving storage and computing time is to restart the algorithm every $k+1$ iterations, using the eurrent estimate for the solution as the new starting guess. Any such method is referred to as a restarled method.

Both of these approaches are discussed by Eisenstat, Elman, and Schultz (1883). Their version of the truncated method is also known as Orthomin(k) (see Vinsome (1976)). The restarted method is known as GClR(k). The special case for $k=0$ is known as the Minimuin llesidual (MlR) method. Work and storage requirements for these methods are presented in Tuble 3.1 . 
Table 1. Work nnd Stornge Requirements for GCR methods.

\begin{tabular}{|c|c|c|c|c|}
\hline & GCR & Orthomin(k) & GCR(k) & $\mathrm{MR}$ \\
\hline Work/Iter & $\begin{array}{c}(3(i+1)+4) n \\
+1 M v\end{array}$ & $\begin{array}{l}(3 k+1) n \\
+1 \mathrm{Mv}\end{array}$ & $\begin{array}{c}((3 / 2) \mathrm{k}+4) \mathrm{n} \\
+1 \mathrm{Mv}\end{array}$ & $\begin{aligned} & 4 \mathrm{n} \\
+ & 1 \mathrm{Mv}\end{aligned}$ \\
\hline Storage & $(2(i+2)+2) n$ & $(2 k+3) n$ & $(2 k+3) n$ & $3 n$ \\
\hline
\end{tabular}

$\mathrm{Mv}=$ Matrix-vector multiply.

\subsection{Convergence Results}

The basic properties of the GCR method are given by Eisenstat, Elman, and Schultz (1983). Since the direction vectors are chosen to be $A^{T} A$-conjugate, a direct argument shows that $x_{i+1}$ minimizes $\left\|r_{i+1}\right\|_{2}$ over Krylov spaces of increasing dimension. Eventually $x_{i+1}$ minimizes the norm of the residual over the whole space. This can be summarized by the following theorem proved by Eisenstat, Elman, and Schultz (1883, Corollary 3.2).

THEOREM 3.1. Let $A$ be an $n \times n$ real matrix such that $M=\left(A+A^{T}\right) / 2$ is positive definite. Then the GCR method gives the exact solution to the system $A x=b$ in at most $n$ iterations.

Although Theorem 3.1 tells us that the GCR method converges in at most $\mathrm{n}$ iterations it does not provide information as to the rate of convergence of the method. The convergence rate is given by the following theorem also proved by Fisenstat, Elman, and Schultz (1983, Theorem 3.3).
THEOREM 3.2. If $A$ is an $n \times n$ real matrix such that $M=\left(A+A^{T}\right) / 2$ is positive definite, and if $\left\{r_{i}\right\}$ is the sequence of residuals generated by GCR, then

$$
\left\|r_{i}\right\|_{2} \leq \min _{9 \in P_{1}}\left\|q_{i}(A)\right\|_{2} \cdot\left\|r_{0}\right\|_{2}
$$

where $P_{i}$ is the class of $i-t h$ degree polynomials. Moreover, if $A$ has a complete set of eigenvectors, and if $J=T^{-1} \Lambda T$ is the Jordan canonical form of $A$, then

$$
\left\|r_{i}\right\|_{2} \leq \kappa(T) \min _{q_{1} \in P,} \max _{\lambda \in \lambda(A)}\left|q_{i}(\lambda)\right| \cdot\left\|r_{0}\right\|_{2}
$$

Theorem 3.2 states that the GCR method is optimal among all polynomial-based iterative methods. Without any information about the structure of the eigenvalues, we cannot pick the best polynomial a priori. However, it can be shown that all of the GCR methods converge using simple properties derived from the iteration process. This convergence proof was provided by Eisenstat, Elman, and Schultz (1983, Theorem 4.1). 
THEOltEM 3.3. If $A$ is an arbitrary real matrix guch that $M=\left(A+A^{T}\right) / 2$ is positive definite, and $R=\left(A^{T}-A\right) / 2$. and if $\left\{r_{i}\right\}$ is the secpuence of residuals generated by GCR, Orthomin(k), GCR(k), or MR then

$$
\left\|r_{1}\right\|_{2} \leq\left[1-\frac{\lambda_{\min }^{2}(M)}{\lambda_{\max }\left(A^{T} A\right)}\right]^{1 / 2}\left\|r_{0}\right\|_{2}
$$

and

$$
\left\|r_{1}\right\|_{2} \leq\left[1-\frac{\lambda_{\operatorname{mat1}}^{2}(M)}{\lambda_{\min }(M) \lambda_{\max }(M)+\rho^{2}(R)}\right]^{i / 2}\left\|r_{0}\right\|_{2}
$$

Elman (1982 p. 141) points out that these bounds are probably not sharp, and his numerical experiments seem to indicate this. We note that if $A$ is symmetric and positive definite so that $R=0$, then the second bound resembles the steepest descent bound (see Luenberger (1073)). This is not too surprising, since the proof for these error bounds is the same for both the GCR method and the MR method. If we don't save any previous directions, that is $k=0$, then all the methods reduce to the MR algorithn which resembles the stcepest descent algorithm.

We also note that Theorem 3.3 does not tell us how to choose $k$. Current folklore is that a value of $k=1$ or $k=2$ provides a $g(x)$ t tradeoll between the work and storagc requirements and an inproved rate of convergence. llowever, this type of analysis is inadequale for determining the effect of the number of saved directions on the rate of convergence. We show in Chapter 4 how to derive a sharper error bound which can be used, in some cases, to determine the optimal number of directions to save.

For the special case of a symmetric operator, the algorithns take on a particularly simple form. An argument parallel to the one used by Eisenstat, Elman, and Schultz (1083 Theorem 4.5) shows the following.

THEOREM 3.4. Let $\mathrm{A}$ be an $n \times n$ symmetric positive definite matrix. Then Orthomin(1) generates the sane iterates as the GCR method.

In essence, Theorem 3.4 states that when the GCK method is applied to a symmetric positive definite matrix the algorithm reduces to the well-known Conjugate Residual Method. This will become important in Chapter 4 when we study the effects of small perturbations to a symmetric operator on the convergence behavior of the GCR algorithm. 


\section{CHAPTER}

\section{The Nonsy mmetric Problem}

This chapter discusses the solution of large, sparse nonsymmetric linear systems. Krylov methods, introduced in Chapter 3 , are discussed in relation to the nonsymmetric problem. First, we review some previous work for nonsymmetric problems. In Section 4.2, we present the standard Chebyshev convergence analysis for the Conjugate Residual method. The main result is presented in Section 4.3. We show that the GCR(k) method converges with a bound which deviates from the error bound for the symmetric case by a term which depends on the size of the nonsymmetry, provided that the method is restarted suficiently often. Section 4.4 treats two applications of our main result for special distributions of eigenvalues.

\subsection{Previous Work}

The Conjugate Gradient method is a popular method for the solution of symmetric positive definite linear systems. However, many important problems give rise to nonsymmetric linear systems, which are usually large and sparse. Therefore, it secms natural to generalize the methods used for the symmetric case to the nonsymmetric case. There are various ways to extend the Conjugate
Gradient method to nonsymmetric systems. Most of these modifications are generalizations of the Conjugate Gradient (CG) method introduced by Hestenes and Stiefel (1052), or the Conjugate Residunl (CR) method developed by Sticel (1955). These methods impose conditions on the iteration method which force certain properties of the Conjugate Gradient method to be satisfied.

Historically, the first suggestion for using the Conjugate Gradient method for general linear systems is due to Hestenes and Stiefel (1952). They suggested using the CG method on the normal equations. If the matrix $A$ has full rank, then the normal equations will be symmetric and positive definite. Fortunately, it is not necessary to form the product $A^{T} A$ since this could lead to a significant loss of precision. Moreover, use of the normal equations has the disadvantage that the convergence rate for conjugate gradients depends on $\kappa\left(A^{T} A\right)$ instead of $\kappa(A)$. If the problem is already moderately ill-conditioned then the resulting iteration scheme could converge slowly.

The Generalized Conjugate Gradient (GCG) metlod developed by Concus and Golub (1976), and by Widlund (1978) was an attempt to modify the CG method to nonsymmetric systems. The GCG method uses a three term recurrence formula for the solution update where certain scalars are chosen to make the residuals of the itcration mutually orthogonal. Consider the itcration

$$
x_{i+1}=x_{i-1}+\omega_{i+1}\left(r_{i}+x_{i}-x_{i-1}\right), \quad i=0,1, \cdots .
$$

If we force the residuals of this iteration to be mutually orthogonal, then we can solve for the scalars $\omega_{i}$. The formulas are given by 


$$
\begin{aligned}
& w_{1}=1 \\
& \omega_{1+1}=\left[1+\frac{\left(11 / \nu_{1}\right)}{\omega_{i}}\right]^{-1,} \quad i=1,2, \cdots,
\end{aligned}
$$

where $\eta_{1} \cdots-\left(r_{1}, r_{1}\right)$

Axelsson (1970) developed a getueralization of the conjugate residual method that difters in the formulas for the solution update. Axelsson computes the stepleng(bs, a, ,by solving the least squares problem:

$$
\min \left\|B^{(i)_{a}^{(i)}}-r_{i}\right\|_{2}
$$

where

$$
B^{(i)} \equiv\left[A p_{0}, \cdots, A p_{i}\right]
$$

The solution to the least syuares problem $(-1.1 .3)$ is equivalent to minimizing the residual at each iteration.

Young and Jea (1080) proposed a moditication, Orthodir, to the CR method. The formula for the direction vectors is replaced with a more expensive calculation to try to improve convergence. In particular, they choose

$$
\begin{gathered}
p_{i+1}=A p_{i+1}+\sum_{j=0}^{i} b(i) p_{i}, \\
b_{j}^{(i)}=\frac{-\left(A^{2} p_{i} A p_{j}\right)}{\left(A p_{j} A p_{j}\right)}
\end{gathered}
$$

Buth Axelsson's method and Orchodir, together with another method proposed by Saad (1083) called GMRES, are mathenatically equivalent to GCR.
They all share the property that at each iteration the residual is mininized over a certain Kírylov subspace.

Sad (1081) used the relationship between the conjugate gradient method and Lanczos (1050) method to develop a class of oblique projection methods. Arnoldi's (1051) method, which is a generalization of the Lanczos method for nonsymmetric systems, is the busie for these projection methods.

Other authors have produced methods not based on the CG method for large nonsymmetric systems. Manteuffel (1977) developed a nonsymmetric version of the Chebyshev method with an adaptive procedure for estimating eigenvalues. The main disadvantage of this method is the need for good estimates of the eigenvalues of the linear operator. These estimates are usually difficult to obtain even for the simplest problems.

Gay (1070) analyzed Broyden's (1065) inethod for linear systems. Although Broyden's method was originally developed for nonlinear systems, Gay showed that for a nonsingular linear system, Bruyden's method converges in at most $2 \mathrm{n}$ iterations for a system of order $n$, and proved that there exist systems for which 2n ilerations are required.

We concentrate on the GCR methods developed by Elman (1982), who sliowed that several versions of the GCR method converge under the assumption that the symmetrie part of the matrix $A$ is positive definite. There are two distinguishing features in our application. The first is that we do not have uccess to the coefficients of the matrix $A$, and so we cannot form $A^{T}$ (sec 
Section 1.2). Unfortunately, in our application we require the vector $A^{T} x$ in all of the above algorithms. The second feature is that the size of the nonsymmetries is small. Elman's analysis predicts a rate of convergence which is too pessimistic in many rases. One would hope that the convergence behavior for our type of problem is similar to the standard CR methods applied to the symmetric problem. We show that for small perturbations to a symmetric operator that the error bound for GCR is not too different from that given by the error bound for CR on the symmetric system. Unfortunately this bound deteriorales as the number of iterations incresses so that we may have to restart the algorithm to obtain an acceptable convergence rate. First we review the standard convergence analysis for the symmetric problem.

\subsection{Convergence Analysis for the Symmetric Problem}

The standard Chebyshev analysis for Conjugate Gradient methods is well known ( see for example Chandra (1078), Cline (1976), Axelsson (1984)) and yields optimal errer bounds for the algorithm. The analysis for both the CG and CR methods is the same, but since we are mainly interested in the GCR incthods we only present the error bounds for the Conjugate Residual method,

Consider the system of linear equations

$$
A x=b
$$

where the matrix $A$ is symmetric and positive definite. Let $P_{k}^{1}$ denote the class of polynomials $p_{k}$ of degree $k$ such that $p_{k}(0)=1$. The following result is due to Chandra (1974, Theorem 3.5).

Theorem 4.1. Let $A$ be a symmetric positive definite matrix. Then for any $k \geq 0$, the iterates of the Conjugate Residual method satisfy

$$
\left\|r_{k}\right\|_{2} \leq \min _{p_{i} \in P_{k}^{1}} \max _{\lambda \in \lambda(\lambda)}\left|p_{k}(\lambda)\right|\left\|r_{0}\right\|_{2}
$$

Theorem 4.1 states that the conjugate residual method generates the optimal polynomial with respect to the $l_{2}$ norm of the residual. The particular error bounds for CIR found in the literature are all derived by considering specific polynomials. For the general case, Engeli, Ginsburg, Rutishauser, and Stiefel (1059) suggest as a candidate poly nomial, $p_{k}$, the one that minimizes the maximum value in an interval containing the spectrum, $\lambda(A)$. The solution using this criterion is given by the normalized Chebyshev polynomial

$$
p_{k}(\lambda)=\frac{T_{k}\left(\frac{2 \lambda-\left(\lambda_{1}+\lambda_{n}\right)}{\lambda_{1}-\lambda_{n}}\right)}{T_{k}\left(\frac{\lambda_{1}+\lambda_{n}}{\lambda_{n}-\lambda_{1}}\right)}
$$

where $T_{k}(z)=\cos (k \arccos z),-1 \leq z \leq 1$. Using the polynomial, $p_{k}(\lambda)$, the following well-known hound enn he derived. Although the proot ean be found in several places (sec for example Cline (1970)) we include it for completeness. 
TuEOnem 1.2. Let $A$ be a symmetric posilive definite matrix. Then for any $k \geq 0$, the iterates of the Conjugate Residual method satisfy the error bound

$$
\left\|r_{k}\right\|_{2} \leq 2\left(\frac{\sqrt{\kappa}-1}{\sqrt{x}+1}\right)^{k}\left\|r_{0}\right\|_{2}
$$

where $\kappa \equiv \kappa(A)=\lambda_{1} / \lambda_{n}$

Proof. Consider the normalized Chebyshev polynomial defined by (4.2.2). Clearly $p_{k}(\lambda) \in P_{k}^{1}$, so that un appplication of Theorem 4.1 yields

$$
\left\|r_{k}\right\|_{2} \leq \max _{\lambda \in \lambda(A)} \frac{\left|T_{k}\left(\frac{2 \lambda-\left(\lambda_{1}+\lambda_{n}\right)}{\lambda_{1}-\lambda_{n}}\right)\right|}{\left|T_{k}\left(\frac{\lambda_{1}+\lambda_{n}}{\lambda_{n}-\lambda_{1}}\right)\right|}\left\|r_{0}\right\|_{2}
$$

Using a property of Chebyshev polynomials that $\left|T_{k}(z)\right| \leq 1,-1 \leq z \leq 1$,

and uoting that $\left|\frac{2 \lambda-\left(\lambda_{1}+\lambda_{n}\right)}{\lambda_{1}-\lambda_{n}}\right| \leq 1(4.2 .4)$ reduces to

$$
\left\|r_{k}\right\|_{2} \leq \frac{1}{T_{k}\left(\frac{\lambda_{1}+\lambda_{n}}{\lambda_{n}-\lambda_{1}}\right)}\left\|r_{0}\right\|_{2}
$$

To bound the term, $T_{k}\left(\frac{\lambda_{1}+\lambda_{n}}{\lambda_{n}-\lambda_{1}}\right)$, consider the $k$-th degree Chebyshev poly nomial,

$$
T_{\Delta}(z)=\cos (k \arccos z) .
$$

From the delinition

$$
\cos (\alpha)=\frac{1}{2}\left(e^{i \alpha}+e^{-i \alpha}\right)
$$

and the relation

$$
e^{i k \theta}=(\cos \theta+i \sin \theta)^{k} \text {, }
$$

equation (4.2.6) can be rewritlen as

$$
T_{k}(z)=\frac{1}{2}\left[\left(z+\sqrt{z^{2}-1}\right)^{k}+\left(z-\sqrt{z^{2}-1}\right)^{k}\right]
$$

For the value $z=\left(\lambda_{1}+\lambda_{n}\right) /\left(\lambda_{n}-\lambda_{1}\right)=(1+\kappa) /(1-\kappa),(4.2 .7)$ becomes

$$
T_{k}\left(\frac{\lambda_{1}+\lambda_{n}}{\lambda_{n}-\lambda_{1}}\right)=\frac{1}{2}\left[\left(\frac{\sqrt{\kappa}-1}{\sqrt{\kappa}+1}\right)^{k}+\left(\frac{\sqrt{\kappa}+1}{\sqrt{\kappa}-1}\right)^{k}\right] \text {, }
$$

where $x$ is the condition number of the matrix A. Combining (4.2.8) and (4.2.5) results in

$$
\left\|r_{k}\right\|_{2} \leq 2\left[\left(\frac{\sqrt{\kappa}-1}{\sqrt{\kappa}+1}\right)^{k}+\left(\frac{\sqrt{\kappa}+1}{\sqrt{\kappa}-1}\right)^{k}\right]^{-1}\left\|r_{0}\right\|_{2} .
$$

Notice that, $\kappa(A) \geq 1$, so that

$$
0 \leq \frac{\sqrt{\kappa}-1}{\sqrt{\kappa}+1}<1<\frac{\sqrt{\kappa}+1}{\sqrt{\kappa}-1}
$$

and hence

$$
\left[\left(\frac{\sqrt{\kappa}-1}{\sqrt{\kappa}+1}\right)^{k}+\left(\frac{\sqrt{\kappa}+1}{\sqrt{\kappa}-1}\right)^{k}\right]^{-1} \leq\left(\frac{\sqrt{\kappa}-1}{\sqrt{\kappa}+1}\right)^{k}
$$

The proof is completed by substituting $(4.2 .10)$ into $(4.2 .8)$. 
The crror hound (4.2.3) depends on two facts, both of which are properties of symmetric matrices: (i) the cigenvalues of the matrix $A$ are known to be real,

(ii) the matrix $A$ is guaranteed to have a complete set of orthogonal eigenvectors and hence is unitarily diagonalizable. Neither one of these two properties is true in general for a nonsymmetric matrix and makes the analysis of Krylov space methods for nonsymmetric matrices more difficult.

In practice, the error bound (4.2.3) can be quite pessimistic for certain problems. Whereas this is the best error bound for the general case, the bound can be improved for special distributions of the eigenvalues of A. Axelsson (1081) derived an improved error bound by assuming that the eigenvalues were distributed over two well separated inlervals of equal length on the positive real axis. Jennings (1977) and Stewart (1075) also obtained results for special distributions of eigenvalues. Jennings considered the effect of one isolated eigenvalue on the convergence rate. Stewart also considered the case of one isolated eigenvalue. However, he concentrated on the convergence rate of the cigenvector associated with the isolated eigenvalue.

\subsection{Perturbational Analysis}

The standard convergence rate analysis for Conjugate Gradient methods is lased on the assumption that the system is symmetric and positive definite. We consider the system

$$
A(\epsilon) x=b,
$$

where

$$
A(\epsilon)=A+\epsilon E, c>0
$$

$A$ is an $n \times n$ symmetric positive definite matrix, and $E$ is a general nonsymmetric matrix such that $\|E\|_{2}=1$.

Elman (1982) has shown that the GCR method generates iterates whose residuals are bounded by

$$
\left\|r_{k}\right\|_{2} \leq \max \left\|q_{k}(A(\epsilon))\right\| \cdot\left\|r_{0}\right\|_{2}
$$

for all polynomials $q_{k}$ of degree $\mathrm{k}$ such that $q_{k}(0)=1$. However without additional information on the structure of the cigenvalues of the matrix $A$ we cannot deduce a general result from this bound. We point out that there exist matrices for which the GCR method converges in no less than $n$ iterations, that is, the residual will not decrease substantially until the last iteration. This point may be clarified by an example. Consider the matrix $\mathrm{J}$ defined by

$$
J=\left[\begin{array}{lllll}
\lambda & \alpha & 0 & . & 0 \\
0 & \lambda & \alpha & . & 0 \\
0 & 0 & \lambda & . & \cdot \\
. & . & . & . & \alpha \\
0 & 0 & 0 & . & \lambda
\end{array}\right]
$$

In this case $\left\|q_{k}(J)\right\|$ is on the order of 1 . In fact, the minimum polynomial for $J$ is the characteristic polynomial (sec Wilkinson (1965) p.41-42). Therefore the GCR method will not produce any substantial decrease until $k=n$. 
In many inportant examples the perturbed matrix is only slightly noisymmetric. lior such systems we would like to consider the effect of small perturbations on the convergence rate of the GCR method. We show that the convergence rate is similar to the standard convergence rate for the CIR method, at leasl for line lirst few iteralions.

We lirst prove a lemna which gives us a bound on the perturbation of a malrix polynomial.

LEMMA 4.3. Let $\phi_{k}(D)$, be a matrix poly nomial of degree $k$, where $D$ is a diagonal matrix, and let $E$ be a general nonsymmetric matrix such that $\|E\|=1$ for some consistent matrix norm. Then

$$
\left\|\phi_{k}(D+\epsilon E)-\phi_{k}(D)\right\| \leq \epsilon \sum_{j=1}^{k} j \cdot\left|c_{k}\right| \cdot(\|D\|+c)^{j-1}
$$

where $c_{k j}$ are the coefficients of the polynomial $\phi_{k}$.

Proof. By the fundarnental theorem of calculus

$$
\phi_{k}(D+\epsilon E)-\phi_{k}(D)=\int_{0}^{\epsilon} d \delta \frac{d \phi_{k}(D+\delta E)}{d \delta}
$$

Taking norms on both sides of (4.3.3) and using Hölder's inequality we obtain

$$
\left\|\phi_{k}(D+\epsilon E)-\phi_{k}(D)\right\| \leq \sup _{0 \leq \delta \leq \varepsilon}\left\|\frac{d \phi_{k}(D+\delta E)}{d \delta}\right\| \cdot\left\|\int_{0}^{\epsilon} d \delta\right\| .
$$

To bound the right-hand side of (4.3.4) consider the derivative term

\section{$\frac{d \phi_{1}(D+\delta E)}{d \delta}$}

ince $\phi_{k}$ is a polynomial of degree $\mathrm{k}$ we can write

$$
\frac{d \phi_{k}(D+\delta E)}{d \delta}=\frac{d}{d \delta} \sum_{j=0}^{k} c_{k j}\left(D+\delta E^{j}\right)^{j}=\sum_{j=0}^{k} c_{k j} \frac{d(D+\delta E)^{j}}{d \delta}
$$

Jsing Liebniz's rule, (4.3.5) can be rewritten as

$$
\frac{d \phi_{k}(D+\delta E)}{d \delta}=\sum_{j=0}^{k} c_{k j} \sum_{i=1}^{j}(D+\delta E)^{i-1} E(D+\delta E)^{j-i}
$$

Taking norms in (4.3.6) and using the triangle inequality yields

$$
\begin{aligned}
\left\|\frac{d \phi_{k}(D+\delta E)}{d \delta}\right\| & \leq\left\|\sum_{j=0}^{k} c_{k j} \sum_{i=1}^{j}(D+\delta E)^{i-1} E(D+\delta E)^{j-i}\right\| \\
& \leq \sum_{j=1}^{k} j \cdot\left|c_{k j}\right| \cdot\|E\| \cdot\|D+\delta E\|^{j-i}
\end{aligned}
$$

Substituting (4.3.7) into (4.3.4) results in the inequality

$$
\left\|\phi_{k}(D+\epsilon E)-\phi_{k}(D)\right\| \leq \sup _{0 \leq \delta \leq e}\left[\sum_{j=1}^{k} j \cdot\left|c_{k j}\right| \cdot\|E\| \cdot\|D+\delta E\|^{j-1}\right] \epsilon .
$$

Notice that

$$
\begin{aligned}
\sup _{0 \leq \delta \leq \epsilon}\|D+\delta E\| & \leq \sup _{0 \leq \delta \leq \epsilon}(\|D\|+|\delta|\|E\|) \\
& \leq\|D\|+\epsilon\|E\| .
\end{aligned}
$$

which we may substitule into ine(guality (4.3.8) to obtain 


$$
\left\|\phi_{k}(D+c E)-\phi_{k}(D)\right\| \leq \epsilon\|E\| \sum_{j=1}^{k} j\left|c_{k j}\right|(\|D\|+c\|E\|)^{j-1} \cdot(4.3 .8)
$$

The proof is completed by using the assumption $\|E\|=1$.

The main result of this study is a bound for the GCR method which applies to the perturbed problem described in equation (4.3.1). We show that when the GCR method is applied to a symmetric operator which has been perturbed, then for the first few itcrations the GCR method generates iterates whose residuals satisfy an error bound that is close to the well-known error bound for the symmetric case.

THEOREM 4.4. Let $A(\epsilon)=A+\epsilon E$, where $A$ is an $n \times n$ symmetric positive definite matrix, and $E$ is an arbitrary matrix such that $\|E\|_{2}=1$. Then the GCR method applied to the perturbed system

$$
A(\epsilon) x=b
$$

yields a sequence of residuals that satisfy the inequality

$$
\frac{\left\|r_{k}\right\|}{\left\|r_{0}\right\|} \leq 2\left(\frac{\sqrt{\kappa}-1}{\sqrt{\kappa}+1}\right)^{k}+r_{k}
$$

where

$$
\tau_{k}=\frac{2 \kappa \delta}{(\sqrt{\kappa}+1)^{k+1}} \sum_{j=1}^{k} j \cdot\left|c_{k j}\right|\left[\frac{\kappa(1+\delta)-1}{\sqrt{\kappa}+1}\right]^{j-1}(\sqrt{\kappa}-1)^{k-j}
$$

$\delta=2 \ell / \lambda_{1}$, and $c_{k j}$ are the coefficients of the $k-t h$ degree Chebysher polynomial.
Prool. Since the matrix $\Lambda$ is symmetric, it is unitnrily dingonalizable, so let $A \equiv D$ be diagonal. From $(4.3 .2)$

$$
\frac{\left\|r_{k}\right\|_{3}}{\left\|r_{0}\right\|_{2}} \leq\left\|q_{k}(D)\right\|_{2}+\left\|q_{k}(D+\epsilon E)-q_{k}(D)\right\|_{2}
$$

The first term on the right hand side of (4.3.10) is exactly the standard convergence rate bound from the symmetric problem. The second term depends on the perturbation and the polynomial chosen. To bound this term we choose a particular polynomial and apply Lemma 4.3 .

By analogy to the symmetric case consider the matrix polynomial

$$
q_{k}(D+\epsilon E)=\frac{T_{k}\left(\frac{2(D+c E)-\left(\lambda_{1}+\lambda_{n}\right) I}{\lambda_{1}-\lambda_{n}}\right)}{T_{k}\left(\frac{\lambda_{1}+\lambda_{n}}{\lambda_{n}-\lambda_{1}}\right)} .
$$

Define

$$
\begin{aligned}
& \hat{D}=\frac{2 D-\left(\lambda_{1}+\lambda_{n}\right) I}{\lambda_{1}-\lambda_{n}} \\
& \hat{c}=\frac{2 \epsilon}{\lambda_{1}-\lambda_{n}} .
\end{aligned}
$$

Then

$$
q_{k}(D+c E)=\frac{T_{k}(\hat{D}+\hat{c} E)}{T_{k}\left(\frac{\lambda_{1}+\lambda_{n}}{\lambda_{n}-\lambda_{1}}\right)}
$$

To bound the second term of (4.3.10) use Lemma 4.3 to yield 


$$
\begin{aligned}
\left\|q_{k}(D+\epsilon E)-q_{k}(D)\right\| & \leq \frac{\left\|T_{k}(\hat{D}+\hat{i} E)-T_{k}(\hat{D})\right\|}{\left|T_{k}\left(\frac{\lambda_{1}+\lambda_{n}}{\lambda_{n}-\lambda_{1}}\right)\right|} \\
& \leq \frac{i \sum_{j=1}^{k} j\left|c_{k}\right| \cdot(\|\hat{D}\|+i)^{j-1}}{\left|T_{k}\left(\frac{\lambda_{1}+\lambda_{n}}{\lambda_{n}-\lambda_{1}}\right)\right|} .
\end{aligned}
$$

By (4.3.12), $\|\dot{D}\|=1$, so that

$$
\left\|q_{k}(D+c E)-q_{k}(D)\right\|_{2} \leq \frac{\frac{2 c}{\lambda_{1}-\lambda_{n}} \sum_{j=1}^{k} j \cdot\left|c_{k j}\right|\left(1+\frac{2 \epsilon}{\lambda_{1}-\lambda_{n}}\right)^{j-1}}{\left|T_{k}\left(\frac{\lambda_{1}+\lambda_{n}}{\lambda_{n}-\lambda_{1}}\right)\right|}
$$

The term in the denominator is bounded ( see Cline (1976)) by

$$
\left|T_{k}\left(\frac{\lambda_{1}+\lambda_{n}}{\lambda_{n}-\lambda_{1}}\right)\right|^{-1} \leq 2\left(\frac{\sqrt{\kappa}-1}{\sqrt{\kappa}+1}\right)^{k}
$$

Substitute (4.3.14) into (4.3.13) and let $\delta=2 £ / \lambda_{1}$. Then (4.3.13) becomes

$$
\left\|q_{k}(D+\epsilon E)-q_{k}(D)\right\|_{2} \leq 2\left(\frac{\sqrt{\kappa}-1}{\sqrt{\kappa}+1}\right)^{k} \frac{\kappa \delta}{\kappa-1} \sum_{j=1}^{k} j \cdot\left|c_{k j}\right|\left(1+\frac{\kappa \delta}{\kappa-1}\right)^{j-1}
$$

where the quantity $\delta$ may be thought of as a normalized error.

Define $\eta_{k}$ by

$$
\eta_{k}=\frac{\kappa \delta}{\kappa-1} \sum_{j=1}^{k} j\left|c_{k j}\right|\left(1+\frac{\kappa \delta}{\kappa-1}\right)^{j-1}
$$

which is a measure of the pertarbation in the Chebyshev matrix polynomial due to the normalized error $\delta$. Then the right liand side of (4.3.15) may be simplitied to

$$
\begin{aligned}
2\left(\frac{\sqrt{\kappa}-1}{\sqrt{\kappa}+1}\right)^{k} \eta_{k} & =\frac{2 \kappa \delta}{(\sqrt{\kappa}+1)^{k+1}} \sum_{j=1}^{k} j \cdot\left|c_{k j}\right|\left[\frac{\kappa(1+\delta)-1}{\sqrt{\kappa}+1}\right]^{j-1}(\sqrt{\kappa}-1)^{k-j} \\
& =\tau_{k}
\end{aligned}
$$

so that $(4.3 .15)$ becomes

$$
\left\|q_{k}(D+\epsilon E)-q_{k}(D)\right\|_{2} \leq \tau_{k}
$$

The proof is completed by substituting (4.3.18) into (4.3.10).

Remark 1. The values of the coefficients $c_{k j}$ of the Chebyshev polynomials are easily computed (see for example Lanczos (1061) p. 455). The coefficients for the lirst 10 Chebyshev polynomials are provided in Table 4.1 .

Remark 2. If $r_{\mathrm{t}}$ is a slowly growing function of $k$ then for the first few iterations we should get a convergence rate similar to the one for the CR method on the unperturbed symmetric problem. A few of the values of $\tau_{k}$ for various values of $\delta$ and $\kappa(A)$ are given in Tables 4.2-4.4. Here we have used the formula

$$
\tau_{k}=2\left[\left(\frac{\sqrt{\kappa}-1}{\sqrt{\kappa}+1}\right)^{k}+\left(\frac{\sqrt{\kappa}+1}{\sqrt{\kappa}-1}\right)^{k}\right]^{-1} \eta_{k},
$$

instead of the expression (4.3.17). For $k \leq 10(4.3 .19)$ provides a tighter bound than the asymptotic formula used in (4.3.17). 
Table 4.1. Chebyshev Cocficients

\begin{tabular}{|c|c|c|c|c|c|c|c|c|c|c|c|}
\hline \multicolumn{12}{|c|}{ Cocfficients for $T_{k}(x)$} \\
\hline \multirow{2}{*}{ k } & \multicolumn{11}{|c|}{$j$} \\
\hline & 0 & 1 & 2 & 3 & 4 & 5 & 8 & 7 & 8 & 9 & 10 \\
\hline 0 & 1 & & & & & & & & & & \\
\hline 1 & 0 & 1 & & & & & & & & & \\
\hline 2 & -1 & 0 & 2 & & & & & & & & \\
\hline 3 & 0 & -3 & 0 & 4 & & & & & & & \\
\hline 4 & 1 & 0 & -8 & 0 & 8 & & & & & & \\
\hline 5 & 0 & 5 & 0 & -20 & 0 & 16 & & & & & \\
\hline B & -1 & 0 & 18 & $\mathbf{0}$ & -48 & 0 & 32 & & & & \\
\hline 7 & 0 & -7 & 0 & 56 & 0 & -112 & $\mathbf{0}$ & 64 & & & \\
\hline 8 & 1 & 0 & -32 & 0 & 160 & 0 & -256 & $\mathbf{0}$ & 128 & & \\
\hline$\theta$ & 0 & $\theta$ & 0 & -120 & 0 & 432 & 0 & -576 & $\mathbf{0}$ & 256 & \\
\hline 10 & -1 & 0 & 50 & $\underline{0}$ & -400 & 0 & 1120 & 0 & -1280 & 0 & 512 \\
\hline
\end{tabular}

Table 4.2. Values of $\tau_{k}$ for Different Condition Numbers Normalized Error $=10^{-6}$

\begin{tabular}{|c|c|c|c|c|}
\hline \multicolumn{5}{|c|}{$\tau_{k}$} \\
\hline \multirow{2}{*}{$\mathrm{c}$} & \multicolumn{5}{|c|}{ Condition Number } \\
\cline { 2 - 5 } & 10 & 100 & 1000 & 10000 \\
\hline \hline 1 & $1.1110^{-6}$ & $1.0110^{-6}$ & $1.0010^{-6}$ & $1.0010^{-6}$ \\
\hline 2 & $4.4410^{-6}$ & $4.0410^{-6}$ & $4.0010^{-6}$ & $4.0010^{-6}$ \\
\hline 3 & $1.6710^{-5}$ & $1.5210^{-5}$ & $1.5010^{-5}$ & $1.5010^{-5}$ \\
\hline 4 & $5.3310^{-5}$ & $4.8510^{-5}$ & $4.8010^{-5}$ & $4.8010^{-5}$ \\
\hline 5 & $1.0110^{-4}$ & $1.4010^{-4}$ & $1.4510^{-4}$ & $1.4510^{-4}$ \\
\hline 6 & $4.6710^{-4}$ & $1.2410^{-4}$ & $4.2010^{-4}$ & $4.2010^{-4}$ \\
\hline 7 & $1.3110^{-3}$ & $1.1010^{-3}$ & $1.1810^{-3}$ & $1.1810^{-3}$ \\
\hline 8 & $3.6310^{-3}$ & $3.3310^{-3}$ & $3.2710^{-3}$ & $3.2610^{-3}$ \\
\hline 0 & $9.8510^{-3}$ & $8.9510^{-3}$ & $8.8710^{-3}$ & $8.8710^{-3}$ \\
\hline 10 & $2.6410^{-2}$ & $2.4010^{-2}$ & $2.3810^{-2}$ & $2.3810^{-2}$ \\
\hline
\end{tabular}

Table 4.3. Values of $r_{k}$ for Different, Condition Numbers Normalized Error $=10^{-3}$

\begin{tabular}{|c|c|c|c|c|}
\hline \multicolumn{5}{|c|}{$\tau_{t}$} \\
\hline \multirow{2}{*}{$k$} & \multicolumn{5}{|c|}{ Condition Number } \\
\cline { 2 - 6 } & 10 & 100 & 1000 & 10000 \\
\hline 1 & $1.1110^{-3}$ & $1.0110^{-3}$ & $1.0010^{-3}$ & $1.0010^{-3}$ \\
\hline 2 & $4.4510^{-3}$ & $4.0410^{-3}$ & $4.0110^{-3}$ & $4.0010^{-3}$ \\
\hline 3 & $1.6710^{-2}$ & $1.5210^{-2}$ & $1.5010^{-2}$ & $1.5010^{-2}$ \\
\hline 4 & $5.3510^{-2}$ & $4.8610^{-2}$ & $4.8210^{-2}$ & $4.8110^{-2}$ \\
\hline 5 & $1.6210^{-1}$ & $1.4710^{-1}$ & $1.4610^{-1}$ & $1.4510^{-1}$ \\
\hline 6 & $4.6810^{-1}$ & $4.2610^{-1}$ & $4.2210^{-1}$ & $4.2210^{-1}$ \\
\hline 7 & $1.3210^{0}$ & $1.2010^{0}$ & $1.1910^{0}$ & $1.1910^{0}$ \\
\hline 8 & $3.6510^{0}$ & $3.3110^{0}$ & $3.2810^{0}$ & $3.2810^{0}$ \\
\hline 9 & $9.9110^{0}$ & $0.0110^{0}$ & $8.9310^{0}$ & $8.9210^{0}$ \\
\hline 10 & $2.6610^{1}$ & $2.4210^{1}$ & $2.4010^{1}$ & $2.3910^{1}$ \\
\hline
\end{tabular}

Table 4.4. Values of $\tau_{k}$ for Different Condition Numbers Normalized Error $=10^{-1}$

\begin{tabular}{|c|c|c|c|c|}
\hline \multicolumn{5}{|c|}{$\tau_{k}$} \\
\hline \multirow{2}{*}{$k$} & \multicolumn{5}{|c|}{ Condition Number } \\
\cline { 2 - 5 } & 10 & 100 & 1000 & 10000 \\
\hline \hline 1 & $1.1110^{-1}$ & $1.0110^{-1}$ & $1.0010^{-1}$ & $1.0010^{-1}$ \\
\hline 2 & $4.9410^{-1}$ & $4.4510^{-1}$ & $4.4010^{-1}$ & $4.4010^{-1}$ \\
\hline 3 & $1.9810^{0}$ & $1.7710^{0}$ & $1.7510^{0}$ & $1.7510^{0}$ \\
\hline 4 & $6.8510^{0}$ & $6.0910^{0}$ & $6.0310^{0}$ & $6.0210^{0}$ \\
\hline 5 & $2.2310^{1}$ & $1.9710^{1}$ & $1.8510^{1}$ & $1.8510^{1}$ \\
\hline 6 & $6.9810^{1}$ & $6.1310^{1}$ & $6.0510^{1}$ & $6.0410^{1}$ \\
\hline 7 & $2.1210^{2}$ & $1.8510^{2}$ & $1.8310^{2}$ & $1.8210^{2}$ \\
\hline 8 & $6.3210^{2}$ & $5.4710^{2}$ & $5.4010^{2}$ & $5.3810^{2}$ \\
\hline 9 & $1.8510^{3}$ & $1.5010^{3}$ & $1.5710^{3}$ & $1.5710^{3}$ \\
\hline 10 & $5.3710^{3}$ & $4.5810^{3}$ & $4.5210^{3}$ & $4.5110^{3}$ \\
\hline
\end{tabular}


An interesting point evident from Tables $4.2-4.4$ is that $\tau_{k}$ approaches a limit as $\kappa(A) \rightarrow \infty$. Using equation $(4.3 .10)$ and taking the linit ay $\kappa(A) \rightarrow \infty$ yieils

$$
\lim _{\kappa \rightarrow \infty} \tau_{k}=\delta \sum_{j=1}^{l} j \cdot\left|c_{k j}\right|(1+\delta)^{j-1}
$$

Unfortunately, the case where the condition number of $A$ is large is not of interest in our application (nor in any practical problem since the CR method would probsbly converge too slowly).

An inmediate consequence of Theorem 4.4 is the special case of a small perturbation to the identity matrix.

COROLLARY 1.5. The GCR method applied to the perturbed system

$$
(I+c E) x=b,\|E\|=1
$$

yields a sequence of residuals that satisfy the inequalities

$$
\frac{\left\|r_{t}\right\|}{\left\|r_{0}\right\|} \leq k \epsilon^{k}
$$

Proof. An application of Theorem 4.4 shows that

$$
\frac{\left\|r_{1}\right\|}{\left\|r_{0}\right\|} \leq r_{k}
$$

Nole that $\kappa(l)=1$, so that $(-1.3 .20)$ is reduces to

$$
\frac{\left\|r_{k}\right\|}{\left\|r_{0}\right\|} \leq \frac{2 \delta}{2^{k+1}} k\left|c_{k t k}\right|\left(\frac{\delta}{2}\right)^{k-1}
$$

The coeflicient $c_{k t}$ in (4.3.21), which is the leading term of the k-th Chebyshev polynuminl $T_{k}(x)$, is

$$
c_{k k}=2^{k-1}
$$

Substituting (4.3.22) into (4.3.21) und using the definition of $\delta=2 c / \lambda_{1}$ completes the prool.

As we already argued in Chapter 3 , the GCik method is really not a practical algorithm for the types of problems we are interested in. Setting aside the issue of storage for the moment, the GCR method is not a practical algorithm because of the large amount of computation needed as the iteration proceeds. Most of this work is in computing the inner products necessary to compute the scalars $b_{j}^{(i)}$, which are used in the calculation of the new direction. In some applications, for example in elliptic partial differential equations, the matrix-vector multiply is not too expensive compared to an inner product, so that as the iteration proceeds it becomes expensive to calculate a new direction. In our application a matrix-vector multiply is defined by the solution of a boundary value problem, so that the inner products are cheap compared to the matrix-vector multiply. Therefore the question of practicality will depend on the specilie application. For the most part, we have also a limited amount of storage so that we are forced to use one of the truncaled or restarted mechods. 
The restarted version of the GCR method, GCR(k), is particuiarly ensy to analyze with the aid of Theorem 4.4.

Recall that the GCR(k) method is the GCR method restarted every $k+1$ iterations. By a cycle we mean any set of residuals generated between any two restarts. For example the $j$-th cycle is

$$
\left\{r_{j(1)}, r_{j(2)}, \cdots, r_{j(k+1)}\right\}
$$

Denote the sequence of residuals generated by the GCR(k) method by

$$
\left\{r_{\alpha(0)}, r_{\alpha(1)}, \cdots, r_{\alpha(k+1)}, r_{1(1)}, \cdots, r_{j(l)}, \cdots\right\}
$$

Notice that

$$
r_{j(0)}=r_{j-1(k+1)} \quad j=1,2, \cdots .
$$

Lei

$$
B_{i}=2\left(\frac{\sqrt{\kappa}-1}{\sqrt{\kappa}+1}\right)^{i}+\tau_{i}
$$

where $\tau_{i}$ is the error term from Theorem 4.4, and $\alpha$ is the condition number of $\boldsymbol{A}$.

We are now in a position to prove an error bound for the GCR(k) method.
THEOREM 4.6. The GCR(k) method applied to the perturbed system

(4.3.1) generales residuals that satisfy the inequalities

$$
\frac{\left\|r_{j(l)}\right\|}{\left\|r_{\alpha(0)}\right\|} \leq B_{l} B_{k+1}^{j}, \quad l=1, \cdots, k+1
$$

Proof. By Theorem 4.4 it follows that within any cycle

$$
\frac{\left\|r_{j(l)}\right\|}{\left\|r_{j(0)}\right\|} \leq B_{l}
$$

Now consider the total reduction in the residual

$$
\frac{\left\|r_{j(l)}\right\|}{\left\|r_{0(0)}\right\|}=\frac{\left\|r_{j(1)}\right\|}{\left\|r_{j(0)}\right\|} \cdot \frac{\left\|r_{j(0)}\right\|}{\left\|r_{\alpha(0)}\right\|}
$$

Using (4.3.23), equation (4.3.25) reduces to

$$
\frac{\left\|r_{j(l)}\right\|}{\left\|r_{\alpha(0)}\right\|}=\frac{\left\|r_{j(l)}\right\|}{\left\|r_{j(0)}\right\|} \cdot \frac{\left\|r_{j-1(k+1)}\right\|}{\left\|r_{\alpha(0)}\right\|} \text {. }
$$

Repeated application of this procedure yields

$$
\frac{\left\|r_{j(l)}\right\|}{\left\|r_{\alpha(0)}\right\|}=\frac{\left\|r_{j(1)}\right\|}{\left\|r_{j(0)}\right\|} \cdot \frac{\left\|r_{j-1(k+1)}\right\|}{\left\|r_{j-1(0)}\right\|} \cdots \frac{\left\|r_{\alpha(k+1)}\right\|}{\left\|r_{\alpha(0)}\right\|}
$$

An application of Theorem 4.4 to each term

$$
\frac{\left\|r_{j(l)}\right\|}{\left\|r_{0(0)}\right\|}=B_{l} \cdot B_{k+1} \quad l=1, \cdots, k+1
$$

completes the proor. 


\subsection{Special Distributions of Eigenvalues}

In this section we discuss two applications of Theorem 4.4 for matrices wilh special distributions of eigenvalues. As in the symmetric case, the error bounc's derived for the GClk method depend on the particular polynomial chosen in Theorem 4.4. The lirst ease we consider is a matrix with eigenvalues that lie in one of two chusters. The sccond case is that of a matrix wilh one isolated large eigenvalue. In both of these cases the theory for the symmetric problem predicts un error bound which is superior to the error bound predicted for the general east (sce Axctison (1084)). The idea in buth cases is to choose a polynomial, $p_{k}(\lambda)$, with $p_{k}(0)=1$, that takes into account the special structure of the spectrum. Using this polynomial, Lemma 4.3 is applied to derive a bound for the maximum of the matrix polynomial over the spectrum of $A$. This bound is then used in place of the standard error bound used for the general case in Theorem 4.4.

Consider the case where the eigenvalues of the matrix $A$ are separated into two distinct clusters of equal width. Let

$$
\lambda(A) \in\left[\lambda_{n}, b\right] \cup\left[c, \lambda_{1}\right],
$$

where $b-\lambda_{n}=\lambda_{1}-c$, and define the polynomial

$$
\dot{P}_{2}(\lambda)=1-\omega \lambda\left(\lambda_{1}+\lambda_{n}-\lambda\right)
$$

If we add the additional constraint that $P_{2}(c)=\mu_{2}\left(\lambda_{1}\right)$, then we can solve for $w$ so that

$$
\tilde{P}_{2}(\lambda)=1-2\left(\lambda_{1}\left(c+\lambda_{n}\right)-\left.c\left(c-\lambda_{n}\right)\right|^{-1} \lambda\left(\lambda_{1}+\lambda_{n}-\lambda\right)\right.
$$

By analogy to the symmetric case consider the polynomial defined by

$$
P_{2}(\lambda)=\frac{T_{1}\left(\frac{2\left(1-\dot{P}_{2}(\lambda)\right)-(\beta+\alpha)}{\beta-\alpha}\right)}{T_{1}\left(\frac{\beta+\alpha}{\alpha-\beta}\right)}
$$

where $\alpha=1-\ddot{P}_{2}\left(\lambda_{n}\right)$, and $\beta=1-\ddot{P}_{2}(b)$. Notice that $P_{2}(0)=1$.

The Chebyshev poly nomial $T_{1}(z)=z$, so that (4.4.3) reduces to

$$
P_{2}(\lambda)=1-\frac{2}{\beta+\alpha}\left(1-\dot{P}_{2}(\lambda)\right)
$$

Substituting (4.4.1) into (4.4.4) results in

$$
P_{2}(\lambda)=1-\frac{2 \omega\left(\lambda_{1}+\lambda_{n}\right)}{(\beta+\alpha)} \lambda+\frac{2 \omega}{(\beta+\alpha)} \lambda^{2}
$$

Applying Lemma 4.3 to the matrix polynomial $P_{2}(D+c E)$, we obtain the inequality

$$
\left.\left\|P_{2}(D+\epsilon E)-P_{2}(D)\right\| \leq \epsilon|| c_{21}|+2| c_{22} \mid(\|D\|+\epsilon)\right]
$$

where $c_{k j}$ are the coefficients of the polynomial $P_{2}(\lambda)$. A straightforward calculation reduces inequality $(\mathbf{4 . 4 . 6 )}$ to

$$
\left\|P_{2}(D+c E)-P_{2}(D)\right\| \leq \frac{\delta}{\beta+\alpha}\left[\frac{(3+\delta) \kappa(A)+1}{1+\left(\frac{1}{\lambda_{1}}+\frac{1}{\lambda_{2}}\right) c-\frac{1}{\lambda_{1} \lambda_{n}} c^{2}}\right]
$$

where $\delta=2 \epsilon / \lambda_{1}$. 
If we use the GCR(1) method then after every 2 iterntions the method will generate a polynomial of degree 2. Therefore, applying Theorem 4.4 with the bound (1.1.7) yields

$$
\frac{\left\|r_{2}\right\|}{\left\|r_{0}\right\|} \leq \frac{1}{\left|T_{1}\left(\frac{\beta+\alpha}{\alpha-\beta}\right)\right|}+\frac{\delta}{\beta+\alpha}\left[\frac{(3+\delta) \kappa(A)+1}{1+\left(\frac{1}{\lambda_{1}}+\frac{1}{\lambda_{a}}\right) c-\frac{1}{\lambda_{1} \lambda_{2}} c^{2}}\right]
$$

Using the definition of the Chebyshev polynomial for $k=1$ results in

$$
\frac{\left\|r_{2}\right\|}{\left\|r_{0}\right\|} \leq \frac{\alpha-\beta}{\beta+\alpha}+\frac{\delta}{\beta+\alpha}\left[\frac{(3+\delta) \kappa(A)+1}{1+\left(\frac{1}{\lambda_{1}}+\frac{1}{\lambda_{2}}\right) c-\frac{1}{\lambda_{1} \lambda_{a}} c^{2}}\right]
$$

Remark. In the special case when $b=\lambda_{n}, c=\lambda_{1}$, then $\alpha=\beta=1$, and (4.4.8) reduces to

$$
\frac{\left\|r_{2}\right\|}{\left\|r_{0}\right\|} \leq \frac{\delta}{4}[(3+\delta) \kappa(A)+1]
$$

The second case of interest is the case of an isolated large eigenvalue. Consider the case where

$$
\lambda \in\left[\lambda_{n}, b\right] \cup\left[\lambda_{1}\right]
$$

The standard error bound involves the condition number of $A$ defined by $\lambda_{1} / \lambda_{n}$. If $\lambda_{1} \gg b$, then this error bound may be a severe overestimate. Cline (1870) has shown that the effect of one isolated large eigenvalue is that of adding one iteration to a problem with a condition number of $\kappa^{\prime}(\Lambda)=b / \lambda_{n}$. We show that the perturbed problem behaves similarly.
Consider the polynominl

$$
P_{k}(\lambda)=\left(1-\frac{\lambda}{\lambda_{1}}\right) \frac{T_{k-1}\left(\frac{2 \lambda-\left(b+\lambda_{n}\right)}{b-\lambda_{n}}\right)}{T_{k-1}\left(\frac{b+\lambda_{n}}{\lambda_{n}-b}\right)}
$$

We seek to bound the term $P_{k}(D+\epsilon E)$, so as in the proof of Theorem 4,4 first write

$$
P_{k}(D+\epsilon E)=P_{k}(D)+\left[P_{k}(D+\epsilon E)-P_{k}(D)\right]
$$

The first term can be bounded by using the standard convergence analysis of Section 4.2. To obtain a bound for the second term of (4.4.11) substitute (4.4.10) and rearrange terms to yield

$$
\begin{aligned}
& P_{k}(D+\epsilon E)-P_{k}(D) \\
& =\left(1-\frac{D}{\lambda_{1}}\right)\left(\frac{T_{k-1}(\dot{D}+\hat{\epsilon} E)-T_{k-1}(\hat{D})}{T_{k-1}\left(\frac{b+\lambda_{n}}{\lambda_{n}-b}\right)}\right)-\frac{\epsilon E}{\lambda_{1}} \frac{T_{k-1}(\hat{D}+\hat{\epsilon} E)}{T_{k-1}\left(\frac{b+\lambda_{n}}{\lambda_{n}-b}\right)}
\end{aligned}
$$

where $\hat{D}$ and $\hat{\epsilon}$ are defined in Section 4.3. Taking norms on both sides of (4.4.12) and noting that $\left\|1-\frac{D}{\lambda_{1}}\right\| \leq 1$, we obtain from (4.4.12)

$$
\begin{aligned}
& \left\|P_{k}(D+\epsilon E)-P_{k}(D)\right\| \\
& \leq \frac{\left\|T_{k-1}(\hat{D}+\hat{\epsilon} E)-T_{k-1}(\hat{D})\right\|}{\left|T_{k-1}\left(\frac{b+\lambda_{n}}{\lambda_{n}-b}\right)\right|}+\frac{\delta}{2} \frac{\left\|T_{k-1}(\hat{D}+\hat{\varepsilon} E)\right\|}{\left|T_{k-1}\left(\frac{b+\lambda_{n}}{\lambda_{n}-b}\right)\right|} .
\end{aligned}
$$


Here again define $\delta=2 e / \lambda_{1}$, and let

$$
\eta_{k-1}=\left\|T_{k-1}(\dot{D}+i \dot{E})-T_{k-1}(\dot{D})\right\| \text {. }
$$

\section{CHAPTER 5}

Then using the triangle incquality and propertics of the Chebyshev polynominls

$$
\begin{aligned}
\left\|T_{k-1}(\hat{D}+\hat{C} E)\right\| & \leq\left\|T_{k-1}(\dot{D})\right\|+\eta_{k-1} \\
& \leq 1+\eta_{k-1} .
\end{aligned}
$$

Substituting (4.4.14) into (4.4.13) yields the bound

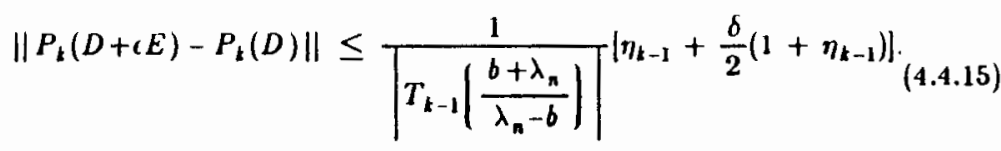

Taking norms on both sides of equation (4.4.11) and using the triangle inequalicy yiclds

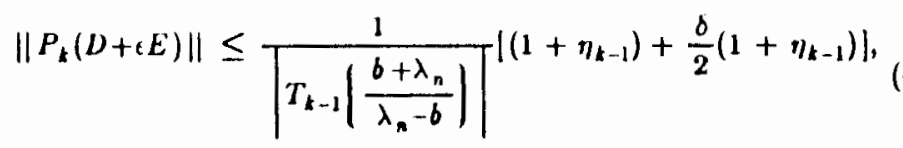

which reduces to

$$
\left\|P_{k}(D+c E)\right\| \leq 2\left(\frac{\sqrt{\kappa^{\prime}}-1}{\sqrt{\kappa^{\prime}}+1}\right)^{k-1}\left[\left(1+\eta_{k-1}\right)+\frac{\delta}{2}\left(1+\eta_{k-1}\right)\right\} \text {, (4.4.17) }
$$

where $\kappa^{\prime}=b / \lambda_{n}$.

As in the symmetric case, the error bound depends on the effective condition mumber $x^{\prime}$. We also note that the eflect of the isoluted large eigenvalue is that of lasing one iteration, if the term $\eta_{t-1}$ is not too large.

\section{Applications and Numerical Resulta}

Phis chapter presents sume numerical results for certain applications of interest. The purpose of these numerical examples is to illustrate some of the important aspects of Theorem 4.4 .

\subsection{Numerical Examples for Small Perturbations}

Theorem 4.A states that for small nonsymetric perturbations to symmetric operators the convergence rate for the $\mathrm{GCR}(\mathrm{k})$ method is similar to the convergence rate for the $\mathrm{CR}$ method applied to the symmetric system. We present several small numerical examples that illustrate this point.

These experiments were run on a Pyramid computer, using double precision arithmetic. The method was said to converge whenever

$$
\frac{\left\|r_{k}\right\|}{\left\|r_{0}\right\|} \leq 10^{-6}
$$

In these test cases, the noise level refers to the size of $t$ in the equation

$$
A(t)=A+t E \text {. }
$$

Sinee the matrix $A$ is symmetric positive definite we assumed that it was already 
diagonalized, so that $A=\operatorname{diag}\left(d_{1}, d_{2}, \cdots, d_{n}\right)$. The nonsymmetric perturbations were generated using the random number generator, URAND, from IMSL. The matrices, E, were computed by generating uniform random numbers between $[-0.5,+0.5]$, and normalizing so that $\|E\|_{2}=1$. The noise level was adjusted by varying $\epsilon$.

The first test case is an application of Corollary 4.5. The matrices in this test case are all of the form $A=I+\epsilon E$. Corollary 4.5 predicts the error bound

$$
\frac{\left\|r_{k}\right\|}{\left\|r_{0}\right\|} \leq k \epsilon^{k}
$$

Tables 5.1-5.2 display the number of iterations required for the GCR(k) method to converge for two matrices of order 10 and 50. Both of these matrices are small perturbations of the identity matrix.

Table 5.1

Perturbation to Identity, $\mathrm{N}=5$.

\begin{tabular}{|c|c|c|c|}
\hline \multicolumn{4}{|c|}{ Number of Iterations } \\
\hline \multirow{2}{*}{$k$} & \multicolumn{3}{|c|}{ Noise Level } \\
\cline { 2 - 4 } & $10^{-6}$ & $10^{-3}$ & $10^{-1}$ \\
\hline 1 & 1 & 2 & 5 \\
\hline 2 & 1 & 2 & 5 \\
\hline 3 & 1 & 2 & 4 \\
\hline 1 & 1 & 2 & 5 \\
\hline 5 & 1 & 2 & 4 \\
\hline
\end{tabular}

Table 5.2

Perturbation to Identity, $\mathrm{N}=50$.

\begin{tabular}{|c|c|c|c|}
\hline \multicolumn{4}{|c|}{ Number of Iterations } \\
\hline \multirow{2}{*}{$k$} & \multicolumn{3}{|c|}{ Noice Level } \\
\cline { 2 - 4 } & $10^{-6}$ & $10^{-3}$ & $10^{-1}$ \\
\hline 1 & 1 & 2 & 5 \\
\hline 2 & 1 & 2 & 5 \\
\hline 3 & 1 & 2 & 5 \\
\hline 4 & 1 & 2 & 5 \\
\hline 5 & 1 & 2 & 5 \\
\hline
\end{tabular}

These results show that the convergence rate is predicted quite well by the theory.

The second set of test cases was chosen to demonstrate the effect of clusters of eigenvalues on the convergence rate. For the symmetric case it is well known that the CR method will converge in at most $m$ iterations, where $m$ is the number of distinct eigenvalues. In fact, the clustering of eigenvalues tends to improve the convergence rate more than would be expected from the standard bound given in Section 3.3. The purpose of this set of tests is to determine if the nunsymmetries would destroy this clustering effect. A secondary goal is to determine if there is an optimal number of directions to save depending on the number of clusters. We ran several enses, with a various number of clustera of eigenvalues. Tables 5.3-5.5 demonstrate the effect of the noise level on the two cluster case. Table 5.6 displays the predicted number of iterations using the theoretical bounds derived in Section 4.4. In the two cluster cascs, the matrix $\Lambda$ 
was formed so that it had two eigenvalues each with multiplicity $n / 2$, that is the matrices are of the form

$$
\begin{aligned}
& d_{1}=\lambda_{1}, \quad 1 \leq i \leq \frac{n}{2} \\
& d_{i}=\lambda_{n}, \quad\left(\frac{n}{2}+1\right) \leq i \leq n
\end{aligned}
$$

Tables 5.7-5.8 present the results for the 3 cluster cases. In the three cluster casc, the matrix A has 3 eigenvalues each with multiplicity $n / 3$. These matrices are of the form

$$
\begin{array}{ll}
d_{i}=\lambda_{1}, & 1 \leq i \leq \frac{n}{3} \\
d_{i}=\lambda_{2}, & \left(\frac{n}{3}+1\right) \leq i \leq \frac{2 n}{3} \\
d_{i}=\lambda_{n}, & \left(\frac{2 n}{3}+1\right) \leq i \leq n
\end{array}
$$

Table 5.3

Two Clusters at $\lambda_{n}=.1, \lambda_{1}=1.0, ; \mathrm{N}=50$.

\begin{tabular}{|c|c|c|c|}
\hline \multicolumn{4}{|c|}{ Number of lerations } \\
\hline \multirow{2}{*}{$k$} & \multicolumn{3}{|c|}{ Noise Level } \\
\cline { 2 - 4 } & $10^{-6}$ & $10^{-3}$ & $10^{-1}$ \\
\hline \hline 0 & 6 & 7 & 60 \\
\hline 1 & 3 & 6 & 22 \\
\hline 2 & 3 & 5 & 20 \\
\hline
\end{tabular}

Table 5.4

Two Clusters at $\lambda_{n}=.01, \lambda_{1}=1.0 . ; \mathrm{N}=50$.

\begin{tabular}{|c|r|r|r|}
\hline \multicolumn{4}{|c|}{ Number of lerations } \\
\hline$k$ & \multicolumn{3}{|c|}{ Norise level } \\
\cline { 2 - 4 } & $10^{6}$ & $10^{3}$ & $10^{1}$ \\
\hline 0 & 4 & 106 & $34^{1}$ \\
\hline 1 & 3 & 8 & $37^{\dagger}$ \\
\hline 2 & 3 & 8 & $56^{\dagger}$ \\
\hline
\end{tabular}

† GCR stalled out (stepsize too small).

Table 5.5

Two Clusters at $\lambda_{n}=.001, \lambda_{1}=1.0, ; \mathrm{N}=50$.

\begin{tabular}{|c|c|r|r|}
\hline \multicolumn{4}{|c|}{ Number of lierations } \\
\hline \hline$k$ & \multicolumn{3}{|c|}{ Noise Level } \\
\cline { 2 - 4 } & $10^{-6}$ & $10^{-3}$ & $10^{-1}$ \\
\hline 0 & 0 & $857^{\dagger}$ & $35^{\dagger}$ \\
\hline 1 & 4 & 16 & $21^{\dagger}$ \\
\hline 2 & 3 & 16 & $32^{\dagger}$ \\
\hline
\end{tabular}

$\dagger$ GClk stalled out (stepsize too small).

Section 4.4 discussed the case where the eigenvalues were contained in two distinct clusters. For the special case where the matrix has only two eigenvalues the analysis predicts the error bound

$$
\frac{\left\|r_{2}\right\|}{\left\|r_{0}\right\|} \leq \frac{\delta}{4}((3+\delta) n(A)+1)
$$

where $\delta=\frac{2 t}{\lambda_{1}}$. Using this error bound, the number of iterations recpuired to reduce the norm of the residual by $10^{6}$ may be computed. If we denote by $p$ the 
minimum number of iterations to reduce the norm of the residual by $10^{-6}$, then

$$
p=2 \cdot \frac{\log \left(10^{-6}\right)}{\log \left[\frac{\delta}{4}((3+\delta) \kappa(A)+1)\right]}
$$

These values are tabulated in Table 5.6. For certain combinations of the condition number and the normalized error the bound in equation (5.1.2) is greater than 1 , so that the predicted number of iterations is meaningless; these values are not displayed. Overall though the predicted number of iterations match very well against the actual number of iterations taken.

Table 5.6

Predicted Number of Iterations for the 2 Cluster Cases.

\begin{tabular}{|c|c|c|c|}
\hline \multicolumn{4}{|c|}{ Number of Iterations } \\
\hline \hline \multirow{2}{*}{ Condition Number } & \multicolumn{3}{|c|}{ Noise Level } \\
\cline { 2 - 4 } & $10^{-6}$ & $10^{-3}$ & $10^{-1}$ \\
\hline \hline 10 & 4 & 7 & $\ldots$ \\
\hline 100 & 4 & 15 & $\ldots$ \\
\hline 1000 & 5 & $\ldots$ & $\ldots$ \\
\hline
\end{tabular}

Table 5.7

Three Clusters at $\mathrm{a}=0.1, \mathrm{~b}=0.5, \mathrm{c}=1.0 ; \mathrm{N}=\mathrm{g}$.

\begin{tabular}{|c|c|c|c|}
\hline \multicolumn{4}{|c|}{ Number of Iterations } \\
\hline \hline$k$ & \multicolumn{3}{|c|}{ Noise Level } \\
\cline { 2 - 4 } & $10^{-6}$ & $10^{-3}$ & $10^{-1}$ \\
\hline \hline 1 & 11 & 11 & 20 \\
\hline 2 & 4 & 7 & 20 \\
\hline 3 & 4 & 7 & 20 \\
\hline 5 & 4 & 6 & 20 \\
\hline
\end{tabular}

Table 5.8

Three Clusters at $a=0.1, b=0.5, c=1.0 ; N=30$.

\begin{tabular}{|c|r|r|r|}
\hline \multicolumn{4}{|c|}{ Number of Itcrations } \\
\hline \multirow{2}{*}{$k$} & \multicolumn{3}{|c|}{ Noise Level } \\
\cline { 2 - 4 } & $10^{-6}$ & $10^{-3}$ & $10^{-1}$ \\
\hline \hline 1 & 11 & 11 & 30 \\
\hline 2 & 4 & 7 & 25 \\
\hline 3 & 4 & 7 & 24 \\
\hline 5 & 4 & 7 & 23 \\
\hline
\end{tabular}

These numerical experiments demonstrate that the GCR(k) method applied to the perturbed problem behaves very much like CR applied to the symmetric problem. In addition these test cases point out that the convergence rate of the GCR(k) method does not improve by saving more directions than is necessary to build up a $k$-th degree polynomial, where $k$ is the number of clusters. For example, the GCR(1) method builds a quadratic polynomial, so that for the two cluster case saving 1 direction is sulficient.

In the third test case, the eigenvalues of the matrix $\mathbf{A}$ are uniformly distributed in the interval $\left[\lambda_{n}, \lambda_{1}\right]$. This purpose of this test case is to determine the effect of the number of saved directions on the convergence rate. Tables 5.9-5.11 illustrate the effect on the convergence rate for various numbers of saved directions and condition numbers. 
Table 5.0

Evenly Spaced Eigenvalues $\in[1.0,10.0] ; N=10$.

\begin{tabular}{|c|c|c|c|}
\hline \multicolumn{3}{|c|}{ Number of lierations } \\
\hline $\mathrm{k}$ & \multicolumn{3}{|c|}{ Noise Level } \\
\cline { 2 - 4 } & $10^{6}$ & $10^{-3}$ & $10^{-1}$ \\
\hline 1 & 30 & 30 & 30 \\
\hline 2 & 27 & 27 & 27 \\
\hline 3 & 23 & 23 & 23 \\
\hline 4 & 10 & 10 & 10 \\
\hline 5 & 21 & 21 & 21 \\
\hline
\end{tabular}

Number of iterations for CR on symmetric problem $=10$.

Tuble 5.10

Evenly Spaced Eigenvalues $\in[1.0,10.0] ; N=50$.

\begin{tabular}{|c|c|c|c|}
\hline \multicolumn{4}{|c|}{ Number of lterations } \\
\hline \multirow{2}{*}{$k$} & \multicolumn{3}{|c|}{ Noise Level } \\
\cline { 2 - 4 } & $10^{-6}$ & $10^{-3}$ & $10^{-1}$ \\
\hline 1 & 31 & 31 & 31 \\
\hline 2 & 26 & 26 & 26 \\
\hline 3 & 24 & 24 & 24 \\
\hline 4 & 23 & 23 & 23 \\
\hline 5 & 22 & 22 & 22 \\
\hline 10 & 21 & 21 & 21 \\
\hline
\end{tabular}

Number of ilerations for CR on symmetric problem $=20$.
Table 5.11

Evenly Spaced Eigenvalues $\in[1.0,100.0] ; N=50$.

\begin{tabular}{|c|r|r|r|}
\hline \multicolumn{4}{|c|}{ Number of Iterations } \\
\hline \multirow{2}{*}{$k$} & \multicolumn{3}{|c|}{ Noise level } \\
\cline { 2 - 4 } & $10^{6}$ & $10^{-3}$ & $10^{-1}$ \\
\hline 1 & 203 & 203 & 201 \\
\hline 2 & 143 & 143 & 143 \\
\hline 3 & 114 & 114 & 113 \\
\hline 4 & 97 & 07 & 97 \\
\hline 5 & 85 & 85 & 86 \\
\hline 10 & 67 & 67 & 66 \\
\hline
\end{tabular}

Number of iterations for CR on symmetric problem $=34$

The number of iterations required for the CR method to converge on the symmetric problem is also given for comparison. These results can also be compared with the predicted number of iterations for the symmetric case. Using the standard Chebyshev bound (see Section 4.2), the predicted number of iterations to reduce the initial norm of the residual by $10^{-6}$ is 23 for a matrix with a $\kappa(A)=10$, and 73 for a matrix with a $\kappa(A)=100$.

In these test cases the noise level does not affect the convergence behavior of the GCR(k) method. This phenomenon can be explained as follows. The unperturbed matrix $A$ bas all simple eigenvalues. In this case, the eigenvalues and eigenvectors of $A$ are both continuous functions of the perturbation (see Wilkinson (1065)). Furthermore, the noise level is small enough that the perturbed matrix also has all simple eigenvalues. Therefore, the inatrix $A(c)$ has a complete set of eigenvectors. Applying Theorem 3.2, we obtain the bound 


$$
\left\|r_{i}\right\|_{2} \leq \kappa(T) \min _{q_{i} \in P_{1}} \max _{\lambda \in \lambda(\lambda)}\left|q_{i}(\lambda)\right| \cdot\left\|r_{0}\right\|_{2}
$$

where $T$ is the matrix whose columns are the eigenvectors of $A$. The matrix $T$ is a perturbation of an orthogonal matrix since $A$ is symmetric, which implies that $T$ is probably well conditioned. Therefore, as long as the noise level is not larger than half the separation distance between eigenvalues, the matrix $T$ should remain well-conditioned, which implies that the bound in (5.1.3) should not change by much.

The fourth test case investigates the effect of an isolated large eigenvalue on the convergence rate. In the case of an isolated large eigenvalue the condition number of the linear system may predict a convergence rate much larger than the one observed. As Cline (1976) has shown, the convergence rate really depends on the effective condition number, that is the condition number of the matrix if the large isolated eigenvalue were removed. The purpose of this test case is to find out if this property is preserved for the case of a small nonsymmetric perturbation. In this test case the matrix $A$ has $n-1$ uniformly distributed eigenvalues in $[1,10]$, and 1 eigenvalue at 100 . Thus the condition number of $\mathrm{A}$ is equal to 100 , but the effective condition number is equal to 10 . Tables 5.12 shows that the isolnted large eigenvalue does slow down the convergence rate, but only as expected from the analysis for the symmetric case. Compring Tables 5.10-5.12 we see that the test ease with the isolated large eigenvalue (Table 5.12) is converging at almost the same rate as the test case with a condition number $=10$ (Table 5.10$)$. This effect is even more pronounced as the number of saved directions increases.

Table 5.12

One Isolated Large Eigenvalue at $\lambda=100 ; N=50$.

\begin{tabular}{|r|c|c|c|}
\hline \multicolumn{4}{|c|}{ Number of Iterations } \\
\hline \hline \multirow{2}{*}{$k$} & \multicolumn{3}{|c|}{ Noise Level } \\
\cline { 2 - 4 } & $10^{-6}$ & $10^{-3}$ & $10^{-1}$ \\
\hline 1 & 50 & 50 & 55 \\
\hline 2 & 35 & 35 & 41 \\
\hline 3 & 28 & 24 & 30 \\
\hline 4 & 25 & 27 & 28 \\
\hline 5 & 23 & 24 & 24 \\
\hline 10 & 21 & 21 & 21 \\
\hline
\end{tabular}

The last test cases use a small variation of Jordan blocks. In these test cases the matrix $A$ is formed by setting the diagonal elements equal to 1 , and the superdiagonal elements equal to $\alpha$, that is,

$$
J=\left[\begin{array}{lllll}
\lambda & \alpha & 0 & . & 0 \\
0 & \lambda & \alpha & . & 0 \\
0 & 0 & \lambda & . & \cdot \\
. & . & . & \cdot & \alpha \\
0 & 0 & 0 & . & \lambda
\end{array}\right]
$$

This is an extreme case of a nonsymmetric matrix In the sense that it has exactly 1 eigenvector regardless of the size of the matrix. Table 5.13 records the results for the test cases where $\alpha=0.1$, and $\alpha=0.5$. These test matrices produce results very similar to test case 1 , where we had small perturbations to 
the identily untrix.

Table: 5.13

Number of Iterations to Converge versus $\alpha ; N=10$.

\begin{tabular}{|c|c|c|}
\hline \multicolumn{3}{|c|}{ Number of lecrutions } \\
\hline \multirow{2}{*}{$k$} & \multicolumn{2}{|c|}{$\alpha$} \\
\cline { 2 - 3 } & 0.1 & 0.5 \\
\hline 1 & 6 & 15 \\
\hline 2 & 6 & 14 \\
\hline 3 & 6 & 14 \\
\hline 4 & 6 & 13 \\
\hline 5 & 6 & 13 \\
\hline 10 & 6 & 10 \\
\hline
\end{tabular}

Table 5.14 displays the results for $\alpha=1$, and various dimensions. The last row of this table displays the number of iterations necessary to converge using the standard CR algorithin, which in this case is equivalent to Orthomin(1). These result verify that taking more directions does not necessarily improve the convergence rate. Anotber point to notice is that the convergence rate does not improve substantially until we use the GCR(n) method.
Table 5.1 .1

Number of Jlerations to Converge for $\alpha=1$, Jordun Blocks.

\begin{tabular}{|c|c|c|c|c|}
\hline \multicolumn{5}{|c|}{ Number or lerations } \\
\hline \hline $\mathrm{k}$ & \multicolumn{4}{|c|}{$\mathrm{N}$} \\
\cline { 2 - 5 } & 5 & 10 & 20 & 50 \\
\hline 1 & 26 & 41 & 63 & 110 \\
\hline 2 & 20 & 40 & 67 & 126 \\
\hline 3 & 33 & 58 & 71 & 133 \\
\hline 4 & 5 & 50 & 76 & 135 \\
\hline 5 & 5 & 54 & 75 & 146 \\
\hline 10 & - & 10 & 81 & 154 \\
\hline $\mathrm{CR}$ & 26 & 41 & 66 & 140 \\
\hline
\end{tabular}

In Table 5.15, we tabulate various properties of the lest matrices which can be used to predict the rate of convergence.

Table 5.15

Spectral Properties of Jordan Blocks.

\begin{tabular}{|l|c|c|c|c|c|}
\hline$\alpha$ & $\mathrm{N}$ & $\|\mathrm{A}\|$ & $\kappa(A)$ & $\|R\|$ & $\delta$ \\
\hline \hline 0.1 & 10 & 1.000 & 1.21 & 0.006 & 0.18 \\
\hline 0.1 & 50 & 1.100 & 1.22 & 0.100 & 0.18 \\
\hline 0.5 & 10 & 1.480 & 2.84 & 0.480 & 0.64 \\
\hline 0.5 & 50 & 1.400 & 2.09 & 0.409 & 0.67 \\
\hline \hline 1.0 & 5 & 1.866 & 13.03 & 0.866 & 0.02 \\
\hline 1.0 & 10 & 1.060 & 48.37 & 0.060 & 0.02 \\
\hline 1.0 & 20 & 1.080 & 178.1 & 0.080 & 1.00 \\
\hline 1.0 & 50 & 2.0 & 1054 & 1.000 & 1.00 \\
\hline
\end{tabular}

llere $\delta$ is the normalized error given by $2\|R\| /\|A\|$.

Nolice that for the cases with $\alpha=1$, the condition number of the symmetric part of $\mathrm{A}$ is close to 1, which implies lhat the Gicle(k) method 
applied to this test case should behave similarly to the test cases where the GCR(k) method was applied to small perturbations of the identity matrix. For the cases with $\alpha=0.5$, the bounds in Tables $4.2-4.6$ predict that $\operatorname{GCR}(2)$ is probably optimal, since the error bounds $B_{k}$ achieve a minimum for $k=2$. Unfortunately the cases with $\alpha=1.0$ yield error bounds greater than 1.0 , so that we cannot use Tables $4.2-4.6$ to predict a convergence rate.

\section{CHAPTER B}

Conclusions

In this study we analyze the behavior of conjugate residual methods for almost symmetric linear systems. The conjugate residual method, which is a popular method for the solution of symmetric positive definite systems is presented and shown to have a convergence rate which depends on the $\sqrt{\kappa(A)}$. We also present the GCR methods, proposed by Eisenstat, Elman, and Schultz. Their convergence rate for the GCR methods depends on the $\kappa(A)$, and is similar to the steepest descent bound. The main result of this study is a new. convergence theorem for the application of the GCR methods to almost symmetric linear systems. This theorem shows that the GCR methods have a convergence rate for the perturbed problem which is a small perturbation of the convergence rate for the $\mathrm{CR}$ method applied to the unperturbed problem. We also give several applications for special distributions of eigenvalues, which show that the GCR methods on the perturbed problem behave similarly to the CR method on the symmetric problem. In addition, some of the analysis indicates that the clustering of the eigenvalues determines how many previous directions to save in the GCR(k) methods. 
There are seill exme questions left unangwered. The analysig used in llis study is a perturbational analysis, and like most analyses of this type it works best for small perturbations. We remark that for large perturbations the error bounds predicted by the theory are meaningless. There is still a question of whether the error bounds derived in this study can be sharpened for large perturbations. Anuther interesting question relates to roundoft crror. Since roundolf error may be considered a small nonsymmetric perturbation to a symmetric operator, it may be possible to apply this work to develop a roundoff error analysis for the conjugate gradient methods.

\section{BIBLIOGRAPHY}

Arnoldi, W.E. $[1051]$. The principle of minimized iteration in the solution of the matrix eigenvalue problem. Quart. Appl. Math., 9:17-29.

Axelsson, O. [1070]. A generalized conjugate direction metlod and its application on a singular perturbation problem. Proceedings of 8 th Biennial Numerical Analysis Conference held at Dundec, Scolland, June 26-29, 1979. Reproduced in Leclure Notes in Mathematics No. 773, pp. 1-12, Springer-Verlag 1980.

Axelsson, O. (1080). Conjugate gradient type methods for unsymmetric and inconsistent systems of linear equations. Linear Alg. Appl., 29:1-16.

Axelsson, O. and Barker, V.A. [1084]. Finite Elemenl Solution of Boundary Value Problems. Academic Press, Orlando, Florida.

Broyden, C.G. [1965]. A class of methods for solving nonlinear simultaneous equations. Math. Comp., 19:577-598.

Chandra, R. [1078]. Conjugate Gradient Methods for Partial Differential Equations. Doctoral Dissertation, Department of Compuler Science, Yale University. Also available as Research Report No. 129.

Cline, A.K. [1076). Several observations on the use of conjugate gradient methods. ICASE Report 76-22. NASA Langley Research Center, flamplon, Virginic.

Concus, P. and Golub, G.I1. [1076]. A generalized conjugate gradient method for nousymmetric systems of linear equations. Technical Report STANCS-76-535, Department of Computer Science, Stanford University.

Danicl, J.W. [1067]. The conjugate gradient method for linear and nonlinear operator equations. SIAMJ. Numer. Anal, 1:10-26.

Dennis, J.E. Jr., (1084). Privale communication. 
Duff, I.S. [1077|. A survey of sparse matrix research. Proc, IEEE, 65:500-595.

Eisenstat, S.C. [1082). A note on the generalized conjugate gradient method. Technical Report No. 228, Department of Computer Science, Yale University.

Eisenstat, S.C., Elman, H.C., and Schultz, M.H. [1083]. Variational iterative methods for nonsymmetric systems of linear equations. SIAM J. Numer. Anal, 20:315-857.

Elman, H.C. [1082]. Iterative Methods for Large Sparse Nonsymmetric Systems of Linear Equations. Doctoral Disserlation, Department of Computer Science, Yale University. Also available as Research Report No. 220.

Engeli, M., Ginsburg, T., Rutishauser, H. and Stiefel, E. [1950]. Refined iterative methods for computation of the solution and the eigenvalues of self-adjoint boundary value problems. Mitleilungen aus dem Institut für angewandle Mathemalik, Birkhäuser Verlag, Basel, Stullgart.

Fox, L. and Parker I.B. [1068]. Chebyshev Polynomials in Numerical Analysis. Oxford University Press, London.

Gay, D.M. [1970]. Some convergence properties of Broyden's method. SIAM J. Numer. Anal., 16:629-690.

Greenbaum, A. [1081]. Behavior of the conjugate gradient algorithm in finite precision arithmetic. Report UCRL 85752, Laurence Livermore Laboratory, Livermore, California.

Hestenes, M.R. and Stiefel, E. [1952]. Method of conjugate gradients for solving linear systems. J. Res. Nal. Bureau Slandards, 19:109-196.

Jea, K.C. [1082]. Generalized Conjugate Gradient Acceleration of Iterative Methods. Doctoral Dissertation, Department of Mathemalics, University of Texas.

Jennings, A. [1077]. Influence of the eigensalue spectrum on the convergence rate of the conjugate gradient method. J. Inst. Math. Appl., 80:61-7R.

Kato, T. [1082]. A Short Introduction to Perturbation Theory for Linear Operalors. Springer Verlag, New York.
Lanczos, C. [1950]. An iteration method for the solution of the eigenvalue problem of linear differential and integral operalors. J. Res. Nat. Bureau Standards, 15:255-282.

Lanczos, C. [1961]. Applied Analysis. Prentice Hall, Englewood Cliffs, New Jersey.

Luenberger, D.G. [1073]. Introduction to Linear and Nonlinear Programming. Addison-Wesley, Reading, Massachusetts.

Manteuffel, T.A. [1977]. The Tchebyshev iteration for nonsymmetric linear systems. Numer. Math., 28:907-327.

Manteuffel, T.A. [1078]. Adaptive procedure for estimating paramelers for the nonsymmetric Tchebychev iteration. Numer. Math., s1:189-208.

Paige, C.C. [1972]. Computational variants of the Lanczos method for the eigenproblem. J. Inst. Math. Appl., 10:\$7s-s81.

Paige, C.C. [1076]. Error analysis of the Lanczos algorithm for tridiagonalizing a symmetric matrix. J. Inst. Math. Appl., 18:311-919.

Paige, C.C. [1980]. Accuracy and effectiveness of the Lanczos algorithm for the symmetric eigenproblem. Linear Alg. Appl., 94:295-258.

Paige, C.C. and Saunders, M.A. [1075]. Solution of sparse indefinite systems of equations and least squares problems. SIAM J. Numer. Anal, 12:617629

Paige, C.C. and Saunders, M.A. [1082]. LSQR: An algorithm for sparse linear equations and sparse least squares. ACM Trans. Malh. So/l., 8:19-71.

Saad, Y. [1080]. Variations on Arnoldi's method for computing eigenelements of large unsymmetric matrices. Linear Alg. Appl., 94:269-295.

Saad, Y. [1981]. Krylov subspace methods for solving large unsymmetric linear systems. Math. Comp., 97:105-126.

Saad, Y. [1982]. The Lanczos biorthogonalization algorithm and other oblique projection methods for solving large unsymmetric systems. SIAM J Numer. Anal, 19:185-506. 
Saad, Y. [1983]. GMIRES: A generalized minimal residual algorilhm for solving monsymuetric linear systems. Technical Report No. 251, Department of Compuler Science, Yale Universily.

Saad, Y'. and Schultz, M.II. [1085]. Conjugate gradient-like algorithms for solving nonsymetric linear systems. Malh. Comp., 11:117-12

Stewart, G.W. [1975]. The convergence of the method of conjugate gradients at isolated extreme points in the spectrum. Numer. Math, $21: 85-93$

Stiefel, E. |1855|. Relaxationmethoden bester Strategie zur Lösung linearer Gleichungssystems. Comm. Malh. Helv., 29:157-178.

Symes, W.W., [1082]. Computational continuation for solutions of wave equations. Unpublished manuscript.

Symes, W.W., [1985]. Stability properties for the velocity inversion problem. To appear in Proc. of the SEG/SIAM/SPE Symposium, Jouston, Teras, January 1985

Vinsome, P.K.W. (1070). ORTILOMIN - An iterative method for solving sparse sets of simultaneous lincar equations. Proc. Fourth SPE Symposium on Reservoir Simulation, Los Angeles, pp. 1/9-160.

Widlund, $\mathrm{O}$. [1878]. A Lanczos method for a class of nonsymmetric systems of linear equations. SlAM J. Numer. Anol, 15:801-812.

Wilkinson, J.H. [1065]. The Algebraic Eigenvalue Problem. OxTord University Press, London.

Wozniakowski, H. [1980]. Roundoff error analysis of a new class of conjugate gradicnt algorithms. Lin. Alg. Appl., 29:507-529.

Young, D.M. and Jea, K.C. [1080]. Gcneralized conjugate-gradient acceleration of non-aymmetrizable ilerative methods. Linear Alg. Appl., S4:158-198 